\title{
Local and Global Theory of the Moduli of Polarized Calabi-Yau Manifolds
}

Andrey Todorov

\begin{abstract}
In this paper we review the moduli theory of polarized CY manifolds. We briefly sketched Kodaira-Spencer-Kuranishi local deformation theory developed by the author and G. Tian. We also construct the Teichmüller space of polarized CY manifolds following the ideas of I. R. Shafarevich and I. I. Piatetski-Shapiro. We review the fundamental result of E. Viehweg about the existence of the course moduli space of polarized CY manifolds as a quasi-projective variety. Recently S. Donaldson computed the moment map for the action of the group of symplectic diffeomorphisms on the space of Kähler metrics with fixed class of cohomology. Combining this results with the solution of Calabi conjecture by Yau one obtain a very conceptual proof of the existence of the coarse moduli space for a large class of varieties. We follow the approach developed in [24] to study the global properties of the moduli of polarized CY manifolds. We discuss the latest results connecting the discriminant locus in the moduli space of polarized odd dimensional CY manifolds with the Bismut-GilletSoule-Quillen-Donaldson Theory of Determinant line bundles.
\end{abstract}

\section{Introduction}

There are two approaches to the construction of the moduli spaces of the polarized algebraic varieties. The first one is analytic. It is based on the local deformation theory of Kodaira-Spencer-Kuranishi. Once such a theory is developed, one can construct the Teichmüller space. The moduli space then is the quotient of the Teichmüller space by some subgroup of the mapping

2000 Mathematics Subject Classification: 14C30, 14E30, 32J27, 32 Q57.

Keywords: Calabi-Yau manifold, Hilbert schemes, Teichmüller space, moduli space of polarized algebraic variety, Weil-Petersson metric, Hodge metric. 
class group of the algebraic variety. It is difficult to obtain some information on the course moduli space of the polarized varieties from this approach. One needs to know some specific proprieties of the Teichmüller space, for instance whether it is a Stein manifold or domain of holomorphy. Once such facts are established if some natural metric like the Weil-Petersson metric or the Hodge metric has some nice curvature properties, then there is a hope that one can prove that the quotient is quasi-projective.

The other approach to the construction of the moduli space of the polarized algebraic varieties is through Hilbert-Mumford's approach to the geometric invariant theory. The role of the Teichmüller space is replaced by the Grothendieck's theory of the Hilbert schemes. Then the moduli space is the quotient of the Hilbert scheme by the group $\mathbb{P} \mathbb{G} L(N)$ on the stable points. Viehweg proved that for a large class of polarized variety the quotient exists as a quasi-projective variety. This class includes the polarized CY manifold. Recently S. Donaldson computed the momentum map of the action of the group of symplectic diffeomorphisms on the space of Kähler metrics with a fix class of cohomology. Combining Donaldson's result with the famous solution of Calabi conjecture of Yau, one obtain immediately the existence of the coarse moduli space for polarized CY manifolds and algebraic manifolds of general type.

One of the main achievements in the theory of Riemann surfaces was the construction of the Teichmüller spaces of the Riemann surfaces. This project was started by Teichmüller and finished by L. Bers and L. Alfors. The definition of a Teichmüller space implies that the mapping class group of the Riemann surface of a fixed genus acts on it. The quotient is the moduli space. In the case of Riemann surfaces of genus greater or equal to one, it is a well known fact that the Teichmüller space is a domain of holomorphy. The Weil-Petersson metric plays important role in the study of moduli of Riemann surfaces. In the case of Riemann surfaces of genus greater than one it was noticed by H. Mazur that the Weil-Petersson metric is not complete. It was Mumford who proved that the moduli space of Riemann surfaces is a quasi-projective variety. He used geometric invariant theory. Later the same result was proved by Bers by using the Weil-Petersson metric.

In the case when the Riemann surface is an elliptic curve, i.e. of genus 1, then it a well-known fact that the Teichmüller space is the upper half plane and the Weil-Petersson metric in this case is the Poincaré metric.

Very little is know about the Teichmüller space in higher dimensions. In the case of polarized abelian varieties of complex dimension $g \geq 2$, the Teichmüller space is the Siegel upper half plane of genus $g$. In the case of polarized algebraic K3 surfaces, Piatetski-Shapiro and Shafarevich constructed the Teichmüller space of marked polarized algebraic K3 surfaces and proved 
the Global Torelli Theorem. It implies that the Teichmüller space is an open and everywhere dense subset in

$$
\mathfrak{h}_{2,19}:=S O_{0}(2,19) / S O(2) \times S O(19)
$$

The complement to the Teichmüller space in $\mathfrak{h}_{2,19}$ corresponds to algebraic K3 surfaces admitting double rational points.

We define the Teichmüller space of any complex manifold as the quotient of all integrable complex structures on $\mathrm{M}$ by the action of the group of diffeomorphisms isotopic to the identity. We will show that there the Teichmüller space of a Calabi-Yau manifold $\widetilde{T}(\mathrm{M})$ exists and it has a finite number of irreducible components. Each component $\mathcal{T}(\mathrm{M})$ is a non-singular complex manifold.

In this paper we review some recent results obtained by the author and K. Liu, Shing-Tung Yau and K. Zuo about the global properties of the moduli space of CY manifolds. See [46], [24], [47] and [48]. We proved that the Teichmüller space of CY manifolds exists and it is non-singular. Based on a result of Selberg and the local Torelli Theorem, we proved the existence of a subgroup in the mapping class group that fixes the polarization such that the quotient of the Teichmüller space by this subgroup is non-singular one. From here using the theory of the determinant line bundles equipped with the Quillen metric we derived that for polarized odd dimensional CY manifolds the moduli space is quasi-affine.

The Dedekind eta function plays an important role in different branches of mathematics. In this paper we will construct the analogue of the Dedekind eta function for odd-dimensional CY manifolds. One way to define the Dedekind eta function is by computing the regularized determinant in the case of the flat metric on an elliptic curve. It is a well-known fact that the zeta function of the Laplacian of the flat metric is given by

$$
E(s)=\left(\frac{1}{2 \pi}\right)^{2 s} \sum_{(n, m) \neq(0,0)} \frac{1}{|n+m \tau|^{2 s}}
$$

where $\tau \in \mathbb{C}, \operatorname{Im} \tau>0$. The computation of the regularized determinant of the flat metric on an elliptic curve is based on the Kronecker limit formula. It was proved that $E(s)$ has a meromorphic continuation in $\mathbb{C}$ with only one pole at $s=1$. According to the Kronecker limit formula

$$
\exp \left(-\left.\frac{d}{d s} E(s)\right|_{s=0}\right)=(\operatorname{Im} \tau)^{2}|\eta|^{4}
$$

where $\eta$ is the Dedekind eta function. 
In the case of the elliptic curves formula (1.1) has the following interpretation. There exists a $C^{\infty}$ section of the determinant line bundle whose Quillen norm is the exponential of the Ray Singer analytic torsion. Moreover, the existence of such a section implies the existence of a multi valued holomorphic section $\eta^{4}$ of the determinant line bundle whose $L^{2}$ norm is equal to the exponential of the Ray Singer analytic torsion. $\left(\eta^{4}\right)^{6}$ can be realized as a holomorphic section of the $24^{\text {th }}$ power of the determinant line bundle over $\mathbb{P S L}_{2}(\mathbb{Z}) \backslash \mathfrak{h}$. Notice that

$$
6=\#\left(\mathbb{P S L}_{2}(\mathbb{Z}) /\left[\mathbb{P S L}_{2}(\mathbb{Z}), \mathbb{P S L}_{2}(\mathbb{Z})\right]\right)
$$

One of the versions of the proof of the Kronecker limit formula is based on the facts that the logarithm of the regularized determinant of the flat metric on the elliptic curve is the potential for the Poincaré metric and this determinant is bounded as a function on the moduli space. For this proof of the Kronecker limit formula see [18]. In this paper we will generalize this method of proving the existence of the analogue of the Dedekind eta function to the case of odd dimensional CY manifolds.

The most interesting property of the $\eta$ function is that it is related to the algebraic discriminant of the elliptic curve. It will be of considerable interest to see the connections between the analogue of the $\eta$ function of odd dimensional CY manifolds and the Gelfand, Kapranov and Zelevinsky theory of the algebraic discriminant. See [16].

In this review paper we gave proofs of some of the Theorems. Some results are just stated.

\section{Local Deformation Theory of CY manifolds}

We will review the local deformation theory for manifolds with canonical class zero that was developed in [42] and [45].

\subsection{Basic Definitions}

Definition 1 Let $M$ be an even dimensional $C^{\infty}$ manifold. We will say that $M$ has an almost complex structure if there exists a section

$$
I \in C^{\infty}\left(M, \operatorname{Hom}\left(T^{*}, T^{*}\right)\right)
$$

such that

$$
I^{2}=-i d
$$

$T$ is the tangent bundle and $T^{*}$ is the cotangent bundle on $M$. 
This definition is equivalent to the following one:

Definition 2 Let $M$ be an even dimensional $C^{\infty}$ manifold. Suppose that there exists a global splitting of the complexified cotangent bundle

$$
T_{M}^{*} \otimes \mathbb{C}=\Omega_{M}^{1,0} \oplus \Omega_{M}^{0,1}
$$

where

$$
\Omega_{M}^{0,1}=\overline{\Omega_{M}^{1,0}} .
$$

Then we will say that $M$ has an almost complex structure.

We are going to define what it means that the almost complex structure is integrable.

Definition 3 We will say that an almost complex structure is an integrable one if for each point $x \in M$ there exists an open set $U \subset M$ such that we can find local coordinates $z^{1}, \ldots, z^{n}$ such that $d z^{1}, \ldots, d z^{n}$ are linearly independent in each point $m \in U$ and they generate $\left.\Omega^{1,0}\right|_{U}$.

It is easy to see that any complex manifold has an almost integrable complex structure. We will define what is a Beltrami differential. We will see how Beltrami differentials distinguish the different complex structures.

Definition 4 Let $M$ be a complex manifold. Let

$$
\phi \in \Gamma\left(M, \operatorname{Hom}\left(\Omega_{M}^{1,0}, \Omega_{M}^{0,1}\right)\right),
$$

then we will call $\phi$ a Beltrami differential.

Since

$$
\Gamma\left(\mathrm{M}, \operatorname{Hom}\left(\Omega_{\mathrm{M}}^{1,0}, \Omega_{\mathrm{M}}^{0,1}\right)\right) \simeq \Gamma\left(\mathrm{M}, \Omega_{\mathrm{M}}^{0,1} \otimes T_{\mathrm{M}}^{1,0}\right),
$$

we deduce that locally $\phi$ can be written as follows:

$$
\left.\phi\right|_{U}=\sum \phi_{\bar{\alpha}}^{\beta} \overline{d z}^{\alpha} \otimes \frac{\partial}{\partial z^{\beta}} .
$$

From now on we will denote by

$$
A_{\phi}=\left(\begin{array}{cc}
i d & \phi(\tau) \\
\overline{\phi(\tau)} & i d
\end{array}\right) .
$$

We will consider only those Beltrami differentials $\phi$ such that $\operatorname{det}\left(A_{\phi}\right) \neq 0$. 
Definition 5 It is easy to see that the Beltrami differential $\phi$ defines a new almost complex structure operator

$$
I_{\phi}=A_{\phi}^{-1} I A_{\phi}
$$

With respect to this new almost complex structure the space $\Omega_{\phi}^{1,0}$ is defined as follows; if $d z^{1}, \ldots, d z^{n}$ generate $\left.\Omega^{1,0}\right|_{U}$, then

$$
d z^{1}+\phi\left(d z^{1}\right), \ldots, d z^{n}+\phi\left(d z^{n}\right)
$$

generate $\left.\Omega_{\phi}^{1,0}\right|_{U}$ and, moreover we have: $\overline{\Omega_{\phi}^{1,0}} \cap \Omega_{\phi}^{1,0}=0$.

We will formulate a criterion for the Beltrami differential $\phi$ to define an integrable complex structure: The Beltrami differential $\phi$ defines an integrable complex structure on $\mathrm{M}$ if and only if the following equation holds:

$$
\bar{\partial} \phi=\frac{1}{2}[\phi, \phi] .
$$

where

$$
\left.[\phi, \phi]\right|_{U}:=\sum_{\nu=1}^{n} \sum_{1 \leqq \alpha<\beta \leqq n}\left(\sum_{\mu=1}^{n}\left(\phi_{\bar{\alpha}}^{\mu}\left(\partial_{\mu} \phi_{\bar{\beta}}^{\nu}\right)-\phi_{\bar{\beta}}^{\mu}\left(\partial_{\nu} \phi_{\bar{\alpha}}^{\nu}\right)\right)\right) \overline{d z}^{\alpha} \wedge \overline{d z}^{\beta} \otimes \frac{\partial}{d z^{\nu}}
$$

(See [31].)

\subsection{Main Results of Local Deformation Theory of CY Manifolds}

The main result in [45] and [42] is the following Theorem:

Theorem 6 Let $M$ be a $C Y$ manifold and let $\left\{\phi_{i}\right\}$ be a basis of harmonic $(0,1)$ forms with coefficients in $T^{1,0}$ of $\mathbb{H}^{1}\left(M, T^{1,0}\right)$, then the equation

$$
\bar{\partial} \phi=\frac{1}{2}[\phi, \phi]
$$

has a solution in the form:

$$
\phi\left(\tau_{1}, \ldots, \tau_{N}\right)=\sum_{i=1}^{N} \phi_{i} \tau^{i}+\sum_{\left|I_{N}\right| \geqq 2} \phi_{I_{N}} \tau^{I_{N}}
$$

where $I_{N}=\left(i_{1}, \ldots, i_{N}\right)$ is a multi-index,

$$
\begin{gathered}
\phi_{I_{N}} \in C^{\infty}\left(M, \Omega^{0,1} \otimes T^{1,0}\right), \\
\tau^{I_{N}}=\left(\tau^{1}\right)^{i_{1}} \ldots\left(\tau^{N}\right)^{i_{N}}
\end{gathered}
$$

and there exists $\varepsilon>0$ such that

$$
\phi(\tau) \in C^{\infty}\left(M, \Omega^{0,1} \otimes T^{1,0}\right)
$$

for $\left|\tau^{i}\right|<\varepsilon$ for $i=1, \ldots, N$. 
In [45] we proved the following basic fact:

Theorem 7 Let $\omega_{0}$ be a holomorphic $n$-form on the $n$ dimensional $C Y$ manifold $M$. Let $\left\{U_{i}\right\}$ be a covering of $M$ and let

$$
\left\{z_{1}^{i}, \ldots, z_{n}^{i}\right\}
$$

be local coordinates in $U_{i}$ such that

$$
\left.\omega_{0}\right|_{U_{i}}=d z_{1}^{i} \wedge \cdots \wedge d z_{n}^{i} .
$$

Then for each $\tau=\left(\tau^{1}, \ldots, \tau^{N}\right)$ such that $\left|\tau_{i}\right|<\varepsilon$ the forms on $M$ defined as:

$$
\left.\omega_{\tau}\right|_{U_{i}}:=\left(d z_{1}^{i}+\phi(\tau)\left(d z_{1}^{i}\right)\right) \wedge \cdots \wedge\left(d z_{n}^{i}+\phi(\tau)\left(d z_{n}^{i}\right)\right)
$$

are globally defined complex $n$ forms $\omega_{\tau}$ on $M$ and, moreover, $\omega_{\tau}$ are closed holomorphic $n$ forms with respect to the complex structure on $M$ defined by $\phi(\tau)$.

Corollary 8 We have the following Taylor expansion for the form $\omega_{\tau}$ :

$$
\left.\left.\omega_{\tau}\right|_{U_{i}}=\omega_{0}+\sum_{k=1}^{n}(-1)^{\frac{k(k-1)}{2}}\left(\left(\wedge^{k} \phi\right)\right\lrcorner \omega_{0}\right) .
$$

(See [45]). From here we deduce the following Taylor expansion for the cohomology class $\left[\omega_{\tau}\right] \in H^{n}(\mathrm{M}, \mathbb{C})$ :

Corollary 9 We have

$$
\left.\left.\left[\omega_{\tau}\right]=\left[\omega_{0}\right]+\sum\left[\left(\phi_{i}\right\lrcorner \omega_{0}\right)\right] \tau^{i}-\sum\left[\left(\left(\phi_{i} \wedge \phi_{j}\right)\right\lrcorner \omega_{0}\right)\right] \tau^{i} \tau^{j}+O\left(\tau^{3}\right) .
$$

(See [45]). We are going to define the Kuranishi family of CY manifolds of any dimension.

Definition 10 Let $\mathcal{K} \subset \mathbb{C}^{N}$ be the policylinder defined by $\left|\tau^{i}\right|<\varepsilon$ for every $i=1, \ldots, N$, where $\varepsilon$ is chosen such that for every $\tau \in \mathcal{K}$,

$$
\phi(\tau) \in C^{\infty}\left(M, \Omega^{0,1} \otimes T^{1,0}\right),
$$

where $\phi(\tau)$ is defined as in Definition 4. On the trivial $C^{\infty}$ family $M \times \mathcal{K}$, we will define for each $\tau \in \mathcal{K}$ an integrable complex structure $I_{\phi(\tau)}$ on the fibre over $\tau$ of the family $M \times \mathcal{K}$, where $I_{\phi(\tau)}$ was defined in Definition 5. Thus we obtained a complex analytic family

$$
\pi: \mathcal{X} \rightarrow \mathcal{K}
$$

of CY manifold. We will call this family the Kuranishi family.

Definition 11 We introduce a coordinate system $\left(\tau^{1}, \ldots, \tau^{N}\right)$ in $\mathcal{K}$ as in Theorem 6. We call this coordinate system a flat coordinate system. The coordinates $\left(\tau^{1}, \ldots, \tau^{N}\right)$ introduced in [45] are exactly the flat coordinates used in [4]. 


\subsection{Automorphisms of CY Manifolds that act trivially on $H^{n}(\mathbf{M}, \mathbb{Z})$}

\section{Theorem 12 Let}

$$
\pi: \mathcal{X} \rightarrow \mathcal{K}
$$

be the Kuranishi family of a polarized $C Y$ manifold $M=\pi^{-1}\left(\tau_{0}\right), \tau_{0} \in \mathcal{K}$. Suppose that $G$ is a group of holomorphic automorphisms of $M$ such that $G$ acts trivially on $H_{n}(M, \mathbb{Z})$ and preserves the polarization. Then $G$ is a finite group of holomorphic automorphisms of all the fibres of the Kuranishi family.

Proof: Since $\mathrm{G}$ acts trivially on $H^{n}(\mathrm{~N}, \mathbb{Z})$ and fixes the polarization class $L$ then the uniqueness the Calabi-Yau metric that corresponds to $L$ implies that the group $G$ is an isometry with respect to the CY metric. Since on CY manifolds there does not exist global holomorphic vector fields, we can conclude that $G$ is a discrete subgroup of the orthogonal group. The compactness of the orthogonal group implies that $G$ is finite. The local Torelli Theorem implies that

$$
\mathcal{K} \subset \mathbb{P}\left(H^{n}(\mathrm{M}, \mathbb{Z}) \otimes \mathbb{C}\right) .
$$

So $\mathrm{G}$ acts on $\mathcal{K}$ and fixes the point $\tau_{0}$. Since $\mathrm{G}$ acts trivially on $H^{n}(\mathrm{M}, \mathbb{Z})$, then it will act trivially on $\mathcal{K}$. Next we are going to prove that if $g \in G$ is any element of $G$ then it acts as complex analytic automorphism on each $\mathrm{M}_{\tau}=\pi^{-1}(\tau)$ for any $\tau \in \mathcal{K}$. This means that

$$
g^{*}\left(I_{\tau}\right)=I_{\tau}
$$

where

$$
I_{\tau} \in C^{\infty}\left(\mathrm{M}, \operatorname{Hom}\left(T_{\mathrm{M}}^{*}, T_{\mathrm{M}}^{*}\right)\right), I_{\tau}^{2}=-i d+\text { integrability }
$$

is the complex structure operator that defines $\mathrm{M}_{\tau}$ for $\tau \in \mathcal{K} . T_{\mathrm{N}}^{*}$ is the cotangent $C^{\infty}$ real bundle. It is a well known fact that

$$
I_{\tau}=\left(A_{\tau}\right)^{-1} \circ I_{0} \circ A_{\tau},
$$

where

$$
A_{\tau}=\left(\begin{array}{cc}
i d & \phi(\tau) \\
\phi(\tau) & i d
\end{array}\right)
$$

See [45]. Let me recall that

$$
\phi(\tau) \in C^{\infty}\left(\mathrm{M}, \operatorname{Hom}\left(\Omega_{\mathrm{M}}^{1,0}, \overline{\Omega_{\mathrm{M}}^{1,0}}\right)\right) \approx C^{\infty}\left(\mathrm{M}, \Theta_{\mathrm{M}} \otimes \Omega_{\mathrm{M}}^{0,1}\right)
$$

and $\phi(\tau)$ satisfies the equation that guarantees the integrability of $I_{\tau}$ defined by $(2.2)$ :

$$
\bar{\partial} \phi(\tau)=\frac{1}{2}[\phi(\tau), \phi(\tau)] \quad \text { and } \quad(\bar{\partial})^{*} \phi(\tau)=0
$$


$\Theta_{\mathrm{M}}$ is the holomorphic tangent bundle. For all the details see [45]. Here $(\bar{\partial})^{*}$ means the conjugate of the operator $\bar{\partial}$ with respect to the Calabi-Yau metric corresponding to the polarization class $L$. If we prove that for each $g \in G$

$$
g^{*}(\phi(\tau))=\phi(\tau)
$$

then (2.4) implies (2.1) and so it is a complex analytic automorphism of $\mathrm{N}_{\tau}$.

Proof of (2.4): According to [45] if we fix a basis $\phi_{1}, \ldots, \phi_{N}$ of harmonic forms of $\mathbb{H}^{1}\left(\mathrm{M}, \Theta_{\mathrm{M}}\right)$ then the solution of the equations (2.3) are given by the power series

$$
\begin{gathered}
\phi(\tau)=\sum_{i=1}^{N} \phi_{i} \tau^{i}+\sum_{i_{1}+\cdots+i_{N}=m>1} \phi_{i_{1}, \ldots, i_{N}}\left(\tau^{1}\right)^{i_{1}} \times \cdots \times\left(\tau^{N}\right)^{i_{N}}=\sum_{m=1}^{N} \phi_{[m]}(\tau) \\
\phi_{[m]}(\tau)=\sum_{i_{1}+\cdots+i_{N}=m} \phi_{i_{1}, \ldots, i_{N}}\left(\tau^{1}\right)^{i_{1}} \times \cdots \times\left(\tau^{N}\right)^{i_{N}}
\end{gathered}
$$

which satisfies the recurrent relation:

$$
\phi(\tau)=\sum_{i=1}^{N} \phi_{i} \tau^{i}+\frac{1}{2}(\bar{\partial})^{*} \circ \mathbb{G}[\phi(\tau), \phi(\tau)] .
$$

See [45]. Here $\mathbb{G}$ is the Green operator associated with the Laplacian with respect to the $\mathrm{CY}$ metric associated with the polarization class $L$. Notice that since $G$ is the group of isometries of CY metric then for any $g \in G$ the Green operator will be invariant, i.e. $g^{*} \mathbb{G}=\mathbb{G}$.

In [45] it is proved that if $\phi \in \mathbb{H}^{1}\left(\mathrm{~N}, \Theta_{\mathrm{N}}\right)$ is a harmonic form with respect to the CY metric then $\phi\lrcorner \omega_{\mathrm{M}}$ will be a harmonic form of type $(n-1,1)$. This fact together with the fact that $G$ acts trivially $H^{n}(\mathrm{M}, \mathbb{C})$ imply that the group acts trivially on $\mathbb{H}^{1}\left(\mathrm{M}, \Theta_{\mathrm{M}}\right)$. This implies that the linear term of (2.5) satisfies

$$
g^{*}\left(\phi_{[1]}(\tau)\right)=\phi_{[1]}(\tau) .
$$

The proof of the fact that

$$
g^{*} \phi(\tau)=\phi(\tau)
$$

is done by induction on the homogeneity of the terms of the power series (2.5). Form $m=1$ this was done. See (2.7). Since for the higher order terms of (2.5) the relation (2.6) implies

$$
\phi_{[k]}=\sum_{p+q=k}\left[\phi_{[p]}(\tau), \phi_{[q]}(\tau)\right]
$$

and $g^{*}\left(\phi_{[p]}\right)=\phi_{[p]}$ for $p<k$ then we can conclude that $g^{*} \phi_{[k]}(\tau)=\phi_{[k]}(\tau)$ for any $k>0$. So the relation (2.4) is proved. Theorem 12 is proved. 


\section{Weil-Petersson Geometry}

\subsection{Basic Facts about Kähler Metrics}

It is stated in [15] that the following conditions are equivalent:

1. Let $g_{i, \bar{j}}$ be a Kähler metric on a complex manifold M.

2. The $(1,1)$ form $\omega:=\operatorname{Im}(g)$ is closed.

3. The complex structure operator $J_{M}$ on $\mathrm{M}$ is a parallel tensor with respect to the Levi-Cevita connection, i.e. $\nabla J_{\mathrm{M}}=0$.

4. Around any point $m_{0} \in M$ there exist, in an open set $\mathcal{U} \subset M$, holomorphic coordinates $\left\{z^{1}, \ldots, z^{n}\right\}$ such that locally the metric $g$ is given by:

$$
g_{i, \bar{j}}=\delta_{i j}+\frac{1}{4} \sum_{k, l} R_{i \bar{j}, k l} z^{k} \bar{z}^{l}+\ldots
$$

where $R_{i \bar{j}, k \bar{l}}$ is the curvature tensor.

The coordinates $\left\{z^{1}, \ldots, z^{n}\right\}$ will be called flat coordinates with respect to the Kähler metric $g$.

\subsection{A Geometric Construction of the Flat Coordinates of a Kähler Metric}

Let us fix a point $m_{0}$ in the complex Kähler manifold M. Let $T_{m_{0}}$ be the tangent space at the point $m_{0} \in M$. We will consider for the moment the tangent space $T_{m_{0}}$ as a real $2 \mathrm{n}$ dimensional vector space. Let $e_{1} \in T_{m_{0}}$ be a vector of length 1 . We will define

$$
e_{n+1}:=J_{\mathrm{M}} e_{1} .
$$

Let $e_{2}$ be a vector perpendicular to the vectors $e_{1}$ and $e_{n+1}$ and $\left\|e_{2}\right\|=1$. We will define

$$
e_{n+2}:=J_{\mathrm{M}} e_{2} .
$$

Continuing this process we obtain a basis in $T_{m_{0}}$ consisting of vectors

$$
\left\{e_{1}, \ldots, e_{n}, J_{\mathrm{M}} e_{1}=e_{n+1}, \ldots, J_{\mathrm{M}} e_{n}=e_{2 n}\right\}
$$

such that they satisfy (3.1) and $\left.<e_{i}, e_{j}\right\rangle=\delta_{i j}$.

Let $\gamma_{i}(t)$ be geodesics for $|t|<\varepsilon$ with respect to the metric $g$ on $M$ such that $\gamma_{i}(0)=m_{0} \in \mathrm{M}$ and

for $i=1, \ldots, n$.

$$
\left.\frac{d \gamma_{i}(t)}{d t}\right|_{t=0}=e_{i}
$$


Definition 13 We will define two dimensional distributions $\mathcal{D}_{i}$ in the tangent bundle $T(M)$ in a small neighborhood $\mathcal{U}$ of the point $m_{0} \in M . \mathcal{D}_{i}$ are defined by parallel transportation along the geodesics containing the point $m_{0}$ of the two dimensional subspaces $\mathcal{E}_{i}(0) \subset T_{m_{0}, M}$ span by $e_{i}$ and $J_{M} e_{i}$ for $i=1, \ldots, n$.

Theorem 14 Locally around the point $m_{0} \in M$ there exist one dimensional complex manifolds $Z_{i}$ for each $1 \leq i \leq n$ such that

1. For each point $x \in \gamma_{i}(t) \subset Z_{i}$ the tangent space $T_{x, Z_{i}}$ of $Z_{i}$ is equal to $\mathcal{D}_{i}(t)$.

2. $Z_{i}$ is a totally geodesic two dimensional real submanifold.

Proof: See [24].

At the origin of a geodesic coordinate system, the metric has the following Taylor expansion:

$$
g_{i j}=\delta_{i j}+\frac{1}{3} R_{i p, g j} x^{p} x^{q}+O\left(|x|^{3}\right)
$$

where $R_{i, p, q, j}$ is the curvature tensor of $g$. (See Proposition 1.14 on page 8 of the book [33].)

\subsection{Basic Definitions and Properties of the Weil-Petersson Metric}

In [42] and [45] a metric on the Kuranishi space $\mathcal{K}$ was defined. This metric was called the Weil-Petersson metric. We will review the basic properties of the Weil-Petersson metric which were established in [42] and [45]. In [45] we proved the following theorem:

Theorem 15 Let $M$ be a $C Y$ manifold of dimension $n$ and let $\omega_{M}$ be a non zero holomorphic $n$ form on $M$ such that

$$
(-1)^{\frac{n(n-1)}{2}}(\sqrt{-1})^{n} \int_{M} \omega_{M} \wedge \overline{\omega_{M}}=1 .
$$

Let $g$ be a Ricci flat $(C Y)$ metric on $M$. Then the map:

$$
\left.\psi \in L^{2}\left(M, \wedge^{m} T^{1,0}\right) \rightarrow \psi\right\lrcorner \omega_{M} \in L^{2}\left(M, \Omega^{n-m, k}\right)
$$

gives an isomorphism between Hilbert spaces and preserves the Hodge decomposition. 
Corollary 16 We can identify the tangent space $T_{\tau}$ at each point $\tau \in \mathcal{K}$ with $H^{1}\left(M, \Omega^{n-1}\right)$ by using the $\left.\operatorname{map} \psi \rightarrow \psi\right\lrcorner \omega_{M}$.

Definition 17 Let $\psi_{1}, \psi_{2} \in T_{\tau}=\mathbb{H}^{1}\left(M_{\tau}, T_{\tau}^{1,0}\right)$ (the space of harmonic forms with respect to the CY metric g). We will define the Weil-Petersson metric as follows:

$$
\begin{aligned}
\left\langle\psi_{1}, \psi_{2}\right\rangle & \left.:=-(-1)^{\frac{n(n-1)}{2}}(\sqrt{-1})^{n} \int_{M_{\tau}}\left(\psi_{1}\right\lrcorner \omega_{\tau}\right) \wedge \overline{\left.\psi_{2}\right\lrcorner \omega_{\tau}}= \\
- & \left.(-1)^{\frac{n(n-1)}{2}}(\sqrt{-1})^{n}<\left(\psi_{1}\right\lrcorner \omega_{\tau}\right), \overline{\left.\psi_{2}\right\lrcorner \omega_{\tau}}>
\end{aligned}
$$

and $\left\|\omega_{\tau}\right\|^{2}=1$.

Notice that $\langle\psi, \psi\rangle>0$ for $\psi \in \mathbb{H}^{1}\left(\mathrm{M}_{\tau}, T_{\tau}^{1,0}\right)$. Next we are going to review the main properties of the Weil-Petersson metric on the Teichmüller space $\widetilde{T}(\mathrm{M})$. The Weil-Petersson metric is a Kähler metric and it defines a natural connection, namely the Levi-Cevita connection. We will denote the covariant derivatives in direction $\frac{\partial}{\partial \tau^{i}}$ at the tangent space of a point $\tau \in \mathcal{K}$ defined by $\phi_{i}$ by $\nabla_{i}$.

\subsection{Flat Coordinates and Curvature}

In [45] we proved the following theorem:

Theorem 18 In the flat coordinate system

$$
g_{i, \bar{j}}(\tau, \bar{\tau})=\delta_{i, \bar{j}}+\frac{1}{6} R_{i \bar{j}, k \bar{l}} \tau^{k} \bar{\tau}^{l}+O\left(\tau^{3}\right)
$$

the following formulas are true for the curvature:

$$
\begin{gathered}
\left.R_{i \bar{j}, k \bar{l}}=\delta_{i \bar{j}} \delta_{k \bar{l}}+\delta_{i \bar{l}} \delta_{k \bar{j}}+\sqrt{-1} \int_{M}\left(\left(\phi_{i} \wedge \phi_{k}\right)\right\lrcorner \omega_{M}\right) \wedge\left(\overline{\left.\left(\phi_{j} \wedge \phi_{l}\right)\right\lrcorner \omega_{M}}\right)= \\
\left.\left.\delta_{i \bar{j}} \delta_{k \bar{l}}+\delta_{i \bar{l}} \delta_{k \bar{j}}-\left\langle\left(\phi_{i} \wedge \phi_{k}\right)\right\lrcorner \omega_{M},\left(\phi_{j} \wedge \phi_{l}\right)\right\lrcorner \omega_{M}\right\rangle .
\end{gathered}
$$

\subsection{Review of Some Recent Results of Z. Lu and X. Sun}

The formula (3.2) for the curvature of the Weil-Petersson metric shows that its holomorphic sectional curvature is not negative. On the other hand the results of Griffiths and Schmidt combined with the local Torelli Theorem showed that there exists a Hermitian metric on the moduli space of CY manifolds with a non-positive holomorphic sectional curvature bounded away from zero. Recently Z. Lu gave explicit formulas for the Hodge metric and proved that it is Kähler and have a non-positive holomorphic sectional curvature bounded away from zero. See [26]. Lu and Sun constructed a Kähler metric with non positive bisectional curvature on the moduli space of CY manifolds. See [28]. 


\section{Teichmüller Theory of CY Manifolds (See [24].)}

\subsection{Some Definitions}

We will follow the methods developed in [24].

Definition 19 We will define the Teichmüller space $\mathcal{T}(M)$ of a $C Y$ manifold $M$ as follows:

$$
\mathcal{T}(M):=\mathcal{I}(M) / \operatorname{Diff}_{0}(M)
$$

where

$$
\mathcal{I}(M):=\{\text { all integrable complex structures on } M\}
$$

and Diffo $(M)$ is the group of diffeomorphisms isotopic to identity. The action of the group Diff $\left(M_{0}\right)$ is defined as follows; Let $\phi \in$ Diff $(M)$, then $\phi$ acts on integrable complex structures on $M$ by pull back, i.e. if

$$
I \in C^{\infty}(M, \operatorname{Hom}(T(M), T(M))),
$$

then we define $\phi\left(I_{\tau}\right)=\phi^{*}\left(I_{\tau}\right)$.

We will call a pair $\left(\mathrm{M} ; \gamma_{1}, \ldots, \gamma_{b_{n}}\right)$ a marked CY manifold when $\mathrm{M}$ is a CY manifold and $\left\{\gamma_{1}, \ldots, \gamma_{b_{n}}\right\}$ is a basis of $H_{n}(\mathrm{M}, \mathbb{Z}) /$ Tor.

Remark 20 Let $\mathcal{K}$ be the Kuranishi space. It is easy to see that if we choose a basis of $H_{n}(M, \mathbb{Z}) /$ Tor in one of the fibres of the Kuranishi family $\mathcal{M} \rightarrow \mathcal{K}$ then all the fibres will be marked, since as a $C^{\infty}$ manifold $\mathcal{X}_{\mathcal{K}} \approx M \times \mathcal{K}$.

\subsection{The Construction of the Teichmüller Space}

Next we are going to construct a universal family of polarized marked $\mathrm{CY}$ manifolds $\pi: \mathcal{U}_{L} \rightarrow \mathcal{T}_{L}(\mathrm{~N})$ up to the action of the group of complex analytic automorphisms $G$ which acts trivially on $H^{2}(\mathrm{~N}, \mathbb{Z})$ on the fibres. The construction of the family $\pi: \mathcal{U}_{L} \rightarrow \mathcal{T}_{L}(\mathrm{~N})$ of marked polarized $\mathrm{CY}$ manifolds follows the ideas of Piatetski-Shapiro and I. R. Shafarevich (see [36]).

Theorem 21 There exists a family of marked polarized CY manifolds

$$
\pi: \mathcal{U}_{L} \rightarrow \mathcal{T}_{L}(M)
$$

which possesses the following properties:

A. $\mathcal{T}_{L}(M)$ is a smooth manifolds of complex dimension $h^{n-1,1}$.

$\boldsymbol{B}$. The holomorphic tangent space $\Theta_{\tau, \mathcal{T}_{L}(M)}$ at each point $\tau \in \mathcal{T}_{L}(M)$ is naturally embedded into $H^{1}\left(M_{\tau}, \Theta_{M_{\tau}}\right)$ and 
C. Let $\pi_{\mathcal{C}}: \mathcal{Y} \rightarrow \mathcal{C}$ be any complex analytic family of marked polarized $C Y$ manifolds such that there exists a point $x_{0} \in \mathcal{C}$ and the fibre $\left(\pi_{\mathcal{C}}\right)^{-1}\left(x_{0}\right)=M_{x_{0}}$ as a marked polarized $C Y$ manifold is isomorphic to some fibre of the family (4.1). Then there exists a unique holomorphic map of families

$$
\kappa:(\mathcal{Y} \rightarrow \mathcal{C}) \rightarrow\left(\mathcal{U}_{L} \rightarrow \mathcal{T}_{L}(M)\right)
$$

defined up to a biholomorphic map $\phi$ of the fibres which induces the identity map on $H_{n}(M, \mathbb{Z})$. The restriction of the map $\kappa$ on the base $\mathcal{C}$ is unique.

Proof: In this paragraph we will use the following result of Y.-T. Siu. See [10] and [37].

Theorem 22 Let $X$ be an algebraic variety and let $\mathcal{L}$ be an ample line bundle then $\left(\mathcal{K}_{X}\right)^{\otimes 2} \otimes(\mathcal{L})^{\otimes m}$ is very ample for any

$$
m \geq 2+\left(\begin{array}{c}
3 n+1 \\
n
\end{array}\right)
$$

where $n$ is the dimension of $X$.

Let us fix the following data; a complex Kähler manifold $\mathrm{M}$ with a canonical class zero, its cohomology ring $H^{*}(\mathrm{M}, \mathbb{Z})$ over $\mathbb{Z}$, its Chern classed and the polarization class $L \in H^{2}(\mathrm{M}, \mathbb{Z})$ which is the Chern class of an ample line bundle. According to a Theorem of Sullivan there are only finite number of $C^{\infty}$ structures on $\mathrm{M}$ if the real dimension of $\mathrm{M}$ is greater or equal to 5 with the data mentioned above. See [39]. Suppose that $m$ satisfy the inequality (4.3) then from the Riemann-Roch-Hirzebruch Theorem, the fact that for very ample line bundles $\mathcal{L}$ we have $H^{k}(\mathrm{X}, \mathcal{L})=0$ for $k>0$ and Theorem 22 , we can deduce that if we consider a CY manifold with fixed Chern classes $c_{2}, \ldots, c_{n}$, fixed cohomology ring $H^{*}(\mathrm{M}, \mathbb{Z})$ and polarization class $L$, then all these Kähler manifolds with a canonical class zero can be embedded in a fixed projective space $\mathbb{C P}^{k}$. Indeed the Hirzebruch-Riemann-Roch theorem implies that for all Kähler manifolds with canonical class zero with we fixed the cohomology ring $H^{*}(\mathrm{M}, \mathbb{Z})$, fixed Chern classes and the line bundle $\mathcal{L}$ with a fixed ample Chern class $L$ the Euler characteristics:

$$
\chi\left(\mathrm{M}, \mathcal{L}^{m}\right)=\operatorname{dim}_{\mathbb{C}} H^{0}\left(\mathrm{M}, \mathcal{L}^{m}\right)=\int_{\mathrm{M}} \operatorname{Td}(\mathrm{M}) C h\left(\mathcal{L}^{m}\right)
$$

is one and the same polynomial of $m$. Using the theory of Hilbert schemes of Grothendieck in [12], we can conclude that there are a finite number of components of the Hilbert scheme that parametrizes all polarized Kähler manifolds with fixed cohomology ring $H^{*}(\mathrm{M}, \mathbb{Z})$, Chern classes and the polarization class $L$. 
The following result is proved in [17]:

Theorem 23 Let $\mathcal{H}_{L}$ be the Hilbert scheme of non-singular CY manifolds embedded by the linear system $\left|L^{m}\right|$ defined by the polarization class $L$, then $\mathcal{H}_{L}$ is a non-singular quasi-projective variety.

We know from the results in [12] there exists a family of polarized CY manifolds:

$$
\mathcal{Y}_{L} \rightarrow \mathcal{H}_{L}
$$

where $\mathcal{Y}_{L} \subset \mathbb{C P}^{N} \times \mathcal{H}_{L}$. Let $\widetilde{\mathcal{H}}_{L}$ be the universal covering of $\mathcal{H}_{L}$ and let

$$
\widetilde{\mathcal{Y}}_{L} \rightarrow \widetilde{\mathcal{H}}_{L}
$$

be the pullback family of (4.4). It is easy to see that the group $\mathbb{S L}_{N+1}$ acts on $\mathcal{H}_{L}$. So this implies that $\mathbb{S L}_{N+1}$ acts also on $\widetilde{\mathcal{H}}_{L}$. We will need the following Lemma and its Corollary:

Lemma 24 Let $G$ be a subgroup of $\mathbb{S L}_{N+1}$ that fixes a point $\tau_{0}$ in $\widetilde{\mathcal{H}}_{L}$, then $G$ is a finite group of complex analytic automorphisms of the $C Y$ manifold $M_{\tau_{0}}$ and it is a normal subgroup of $\mathbb{S L}_{N+1}$ that acts trivially on $H_{n}(M, \mathbb{Z})$.

Proof: According to Theorem 23, $\mathcal{H}_{L}$ is a smooth quasi-projective variety. So its universal cover $\widetilde{\mathcal{H}}_{L}$ is a simply connected complex manifold and we may suppose that the family (4.5) is marked and polarized. The Definition 20 of the marked family of CY manifolds implies that if $G$ fixes the point $\tau_{0} \in \widetilde{\mathcal{H}}_{L}$, then $G$ is a subgroup in $\mathbb{S L}_{N+1}$ that stabilizes $\mathrm{M}_{\tau_{0}}$ in $\mathbb{C P}^{N}$. This shows that $G$ must be a group of holomorphic automorphisms of $\mathrm{N}_{\tau_{0}}$ and it must act trivially on $H_{n}(\mathrm{M}, \mathbb{Z})$. Theorem 12 implies that $G$ acts trivially on $\widetilde{\mathcal{H}}_{L}$. This will imply that it is a normal subgroup in $\mathbb{S L}_{N+1}$. To show this we need to prove that for any element $g \in \mathbb{S L}_{N+1}$ we have

$$
g^{-1} G g=G \text {. }
$$

Let $\tau=g^{-1}\left(\tau_{0}\right)$. Then direct computations show that for any element $h \in G$ we have

$$
g^{-1} h g(\tau)=\tau .
$$

The last equality shows that $G$ is a normal subgroup. Lemma 24 is proved.

Corollary 25 The group $G_{1}:=\mathbb{S L}_{N+1}(\mathbb{C}) / G$ acts freely on $\widetilde{\mathcal{H}}_{L}$.

We will prove that the quotients $\widetilde{\mathcal{Y}}_{L} / G_{1}$ and $\widetilde{\mathcal{H}}_{L} / G_{1}$ exist as complex manifolds and that

$$
\mathcal{U}_{L}:=\widetilde{\mathcal{Y}}_{L} / G_{1} \rightarrow \mathcal{T}_{L}(N):=\widetilde{\mathcal{H}}_{L} / G_{1}
$$

will be the family (4.1) with the properties stated in the Theorem. 
Palais proved in [32] that if an arbitrary Lie group $\mathfrak{G}$ acts on a complex manifold $\mathrm{Y}$, then the quotient $\mathrm{Y} / \mathfrak{G}$ exists in the category of complex spaces, provided that the map

$$
f: \mathfrak{G} \times \mathrm{Y} \rightarrow \mathrm{Y} \times \mathrm{Y}
$$

defined as $\mathrm{f}(g, m)=(g(m), m)$ is proper. In order to prove that the morphism defined by the action of the Lie group

$$
\mathfrak{G}=G_{1}:=\mathbb{S L}_{k+1}(\mathbb{C}) / G
$$

acts properly on $\widetilde{\mathcal{H}}_{L}$ and $\widetilde{\mathcal{Y}}_{L}$, we need to use the following criterion for the properness of the map that can be found in [11], Chapter II, 7:

Criterion 26 Let $f: X \rightarrow Z$ be a morphism of two complex manifolds, let $\mathcal{D}$ be the unit disk, let $\phi^{*}: \mathcal{D}^{*} \rightarrow X$ be a holomorphic map from the punctured disk to $X$ and let $h: \mathcal{D} \rightarrow Z$ be a holomorphic map such that

$$
f \circ \phi^{*}=h \text {. }
$$

If for such $g$ and $h$ there exists a holomorphic map $\phi ; \mathcal{D} \rightarrow X$ coinciding with $\phi^{*}$ on $\mathcal{D}^{*}$, then the morphism $f$ is proper.

The existence of the Grothendieck families $\widetilde{\mathcal{Y}}_{L} \rightarrow \widetilde{\mathcal{H}_{L}}$ defines the following families

$$
\begin{array}{ll}
\pi_{1}: & \widetilde{\mathcal{N}}_{L} \rightarrow G_{1} \times \widetilde{\mathcal{H}}_{L} \\
\pi_{2} & : \quad \widetilde{\mathcal{R}}_{L} \rightarrow \widetilde{\mathcal{H}_{L}} \times \widetilde{\mathcal{H}_{L}}
\end{array}
$$

where $\pi_{1}^{-1}(g, \tau):=\mathrm{N}_{g(\tau)} \times \mathrm{N}_{\tau}$ and $\pi_{2}^{-1}(g(\tau), \tau)=\mathrm{N}_{g(\tau)} \times \mathrm{N}_{\tau}$.

Next we will apply Criterion 26 to the map $f$ defined by (4.6). The condition (4.7) and the definition of the map $f$ as the action of the group $G_{1}$ on $\widetilde{\mathcal{H}}_{L}$, imply that for the image $g(\mathcal{D})$ of the disk $\mathcal{D}$ we have two possibilities.

I. Let

$$
p_{2}: \widetilde{\mathcal{H}_{L}} \times \widetilde{\mathcal{H}_{L}} \rightarrow \widetilde{\mathcal{H}_{L}}
$$

be the projection on the second factor. The first one is that the second projection $p_{2}\left(f\left(\phi^{*}\left(\mathcal{D}^{*}\right)\right)\right)$ is a punctured disk in $\widetilde{\mathcal{H}_{L}}$. Since $p_{2}\left(f\left(\phi^{*}\left(\mathcal{D}^{*}\right)\right)\right)$ has complex dimension one and the map $f$ is defined as

$$
f(g, \tau)=(g(\tau), \tau)
$$

we can conclude that the first projection $p_{1}\left(\phi^{*}\left(\mathcal{D}^{*}\right)\right) \subset G_{1}$ is a point in $g_{0} \in G_{1}$. Then the condition (4.7) implies directly the existence of $\phi$ which 
is an extension of the map $\phi^{*}$ from the punctured disk to the disk. Indeed this follows directly since the second projection restricted to the second summand is the identity map.

II. The second possibility is that

$$
p_{2}\left(f\left(\phi^{*}\left(\mathcal{D}^{*}\right)\right)\right)=\tau_{0}
$$

is a point. This means

$$
\phi^{*}\left(\mathcal{D}^{*}\right)=\mathcal{D}_{1}^{*} \times \tau_{0} \subset \widetilde{\mathcal{H}}_{L} \times \widetilde{\mathcal{H}}_{L}
$$

where $\mathcal{D}_{1}^{*}$ is a punctured disk in the Lie group $G_{1}$. This means that $p_{1}\left(h\left(\mathcal{D}^{*}\right)\right)$ will be contained in the orbit $G_{1}$ of the point $\tau_{0}$ in $\widetilde{\mathcal{H}}_{L}$. So combining (4.11) with the existence of the families (4.8) and (4.9) one gets that the pullbacks by

$$
h: \mathcal{D} \rightarrow \widetilde{\mathcal{H}}_{L} \times \widetilde{\mathcal{H}}_{L}=\mathrm{Z}
$$

and

$$
\phi^{*}: \mathcal{D}^{*} \rightarrow G_{1} \times \widetilde{\mathcal{H}}_{L}=\mathrm{X}
$$

define families $V \rightarrow \mathcal{D}$ and $W^{*} \rightarrow \mathcal{D}^{*}$ of marked and polarized CY manifolds over the disk and the punctured disk respectively. (4.10) implies that the family of polarized CY manifolds $W^{*} \rightarrow \mathcal{D}^{*}$ is the trivial family, i.e.

$$
W^{*} \approx \mathcal{D}^{*} \times \mathrm{M}
$$

The condition (4.7) implies that the holomorphic map $f$ induces a non trivial map between the families $f^{*}:\left.W^{*} \rightarrow V\right|_{\mathcal{D}^{*}}=V^{*}$ which on each fibre is an isomorphism. So the family $V^{*} \rightarrow \mathcal{D}^{*}$ will be trivial too. Since $\mathrm{M}$ is a $\mathrm{CY}$ manifold and thus is not ruled then Theorem 2 of [29] allows us to conclude that the map $f$ can be prolonged to a map $\bar{f}: W \rightarrow V$ of the trivial families such that its restriction to each fibre is an isomorphisms preserving the polarization. So the restriction of $\bar{f}$ over the zero fibre

$$
\bar{f}_{0}: \mathrm{M} \rightarrow \mathrm{M}
$$

is an isomorphism which preserve the polarization. Now can conclude that $\phi^{*}$ can be prolonged to a holomorphic map

$$
\phi: \mathcal{D} \rightarrow G_{1} \times \widetilde{\mathcal{H}}_{L}
$$

So the action of $G_{1}$ on $\widetilde{\mathcal{H}}_{L}$ is proper. The quotient $\widetilde{\mathcal{H}}_{L} / G_{1}$ exists and it is a smooth algebraic variety. 
The situation is analogous to the morphism

$$
\tilde{\mathcal{Y}}_{L} \rightarrow \mathcal{U}_{L}=\tilde{\mathcal{Y}}_{L} / G_{1}
$$

Thus I proved that the quotients $\widetilde{\mathcal{Y}}_{L} / G_{1}$ and $\widetilde{\mathcal{H}}_{L} / G_{1}$ exist as complex spaces. It is obvious that they define a family

$$
\mathcal{U}_{L} \rightarrow \mathcal{T}_{L}(\mathrm{~N})
$$

of marked polarized CY manifolds. According to Corollary 25, $G_{1}$ acts on $\mathcal{Y}_{L}$ and $\mathcal{H}_{L}$ without fixed points. Therefore,

$$
\widetilde{\mathcal{Y}}_{L} / G_{1}=\mathcal{U}_{L} \text { and } \widetilde{\mathcal{H}}_{L} / G_{1}=\mathcal{T}_{L}(\mathrm{~N})
$$

are manifolds. Thus we constructed the family (4.1). We also proved that the base of the family $(4.1) \mathcal{T}_{L}(\mathrm{~N})$ is smooth. Next we will prove that the complex dimension of $\mathcal{T}_{L}(\mathrm{~N})$ is $h^{n-1,1}$ and that the tangent space at each point $\tau \in \mathcal{T}_{L}(\mathrm{~N})$ is embedded in $H^{1}\left(\mathrm{M}_{\tau}, \Theta_{\mathrm{M}_{\tau}}\right)$.

We shall prove that the family (4.1) is effectively parametrized. According to Kodaira this means that for any point $s \in \mathcal{T}(\mathrm{M})$ the mapping

$$
T_{s, \mathcal{T}_{L}(\mathrm{M})} \rightarrow H^{1}\left(\mathrm{M}_{s}, \Theta_{\mathrm{M}_{s}}\right)
$$

is an isomorphism. From the construction of $\mathcal{T}_{L}(\mathrm{M})$ as defined in (4.15) we can conclude that

$$
T_{s, \mathcal{T}_{L}(\mathrm{M})} \approx H^{0}\left(\mathrm{M}, \mathcal{N}_{\mathbb{C P}^{N} / \mathrm{M}}\right) / \operatorname{sl}(N+1)
$$

where $\operatorname{sl}(N+1)$ is the Lie algebra of the group $\mathbb{S L}(N+1)$. In [17] we proved that

$$
H^{0}\left(\mathrm{M}, \mathcal{N}_{\mathbb{C P}^{N} / \mathrm{M}}\right) / \operatorname{sl}(N+1) \approx H^{1}\left(\mathrm{M}, \Theta_{\mathrm{M}}\right) .
$$

Theorem 23 is proved.

Corollary 27 Let $\mathcal{Y} \rightarrow X$ be any family of marked $C Y$ manifolds, then there exists a unique holomorphic map

$$
\phi: X \rightarrow \mathcal{T}_{L}(M)
$$

up to a biholomorphic map $\psi$ of $M$ which induces the identity map on $H_{n}(M, \mathbb{Z})$.

From now on we will denote by $\mathcal{T}(\mathrm{M})$ the irreducible component of the Teichmüller space that contains our fixed CY manifold M. 


\subsection{Global Properties of the Teichmüller Space}

We will conjecture that

Conjecture 28 There exists a normal subgroup $\Gamma_{0}$ in the mapping class group $\Gamma(M)$ of a $C Y$ manifold such that $\mathcal{T}(M) / \Gamma_{0}$ can be realized as an open and everywhere dense subset in a domain of holomorphy $\mathfrak{D}(M)$ embedded in $H^{1}\left(M, T_{M}^{1,0}\right)$. The complement of $\mathcal{T}(M) / \Gamma_{0}$ in $\mathfrak{D}(M)$ is a countable union of closed analytic subvarieties.

Conjecture 28 can be considered as the analogue of the epimorphism Theorem for polarized algebraic K3 surfaces. In case of marked polarized K3 surfaces the Teichmüller space is an open and everywhere dense subset in the symmetric domain $S O_{0}(2,19) / S O(2) \times S O(19)$. The complement to it consists of countable union of divisors and the points of the complement correspond to K3 surfaces with double rational points. In case of CY manifolds the points of the complement to $\mathcal{T}(\mathrm{M}) / \Gamma_{0}$ in $\mathfrak{D}(\mathrm{M})$ correspond to singular manifolds such that after we resolved the singularities on them we obtain a non singular manifold with a holomorphic $n$ form which has zeros.

It seems possible that Conjecture 28 implies the analogue of the global Torelli Theorem for the Teichmüller space. Global Torelli Theorem fails for the moduli space of polarized CY manifolds. Balàzs Szendroi constructed counter examples to the Global Torelli Theorem for the case when one considers the period map from moduli space of polarized CY manifolds to period domain. See [40] and [41].

\section{Moduli of CY Manifolds (See [24])}

\subsection{Application of a Theorem of Selberg to the Moduli of CY Manifolds}

Definition 29 We will define the mapping class group $\Gamma(M)$ of any compact $C^{\infty}$ manifold $M$ as follows: $\Gamma=\operatorname{Diff}_{+}(M) / \operatorname{Diff}_{0}(M)$, where $\operatorname{Diff}_{+}(M)$ is the group of diffeomorphisms of $M$, preserving the orientation of $M$, and $\operatorname{Diff}_{0}(M)$ is the group of diffeomorphisms isotopic to identity.

Definition 30 Let $L \in H^{2}(M, \mathbb{Z})$ be the imaginary part of a Kähler metric. Let

$$
\Gamma_{L}:=\left\{\phi \in \Gamma_{L} \mid \phi(L)=L\right\}
$$

It is a well known fact that the moduli space of polarized algebraic manifolds $\mathcal{M}_{L}(\mathrm{M})=\mathcal{T}(\mathrm{M}) / \Gamma(\mathrm{M})$. 
Theorem 31 There exists a subgroup of finite index $\Gamma$ of $\Gamma(M)$ such that $\Gamma$ acts freely on $\mathcal{T}(M)$ and $\Gamma \backslash \mathcal{T}(M)=\mathfrak{M}(M)$ is a non-singular quasi-projective variety.

Proof of Theorem 31: In the case of odd dimensional CY manifolds there is a homomorphism induced by the action of the diffeomorphism group on the middle homology with coefficients in $\mathbb{Z}$ :

$$
\phi: \Gamma(\mathrm{M}) \rightarrow \mathbb{S p}\left(2 b_{n}, \mathbb{Z}\right) .
$$

In the case of even dimensional CY, there is a homomorphism

$$
\phi: \Gamma(\mathrm{M}) \rightarrow \mathbb{S O}(2 p, q ; \mathbb{Z}) .
$$

$\mathrm{SO}(2 p, q ; \mathbb{Z})$ is the group of the automorphisms of the lattice $H_{n}(\mathrm{M}, \mathbb{Z}) /$ Tor. An important theorem due to Sullivan, proved in [39], states:

Theorem 32 Suppose that the real dimension of a $C^{\infty}$ manifold $M$ is bigger or equal to 5, then the image $\phi(\Gamma(M))$ of the mapping class group is an arithmetic group.

This result of Sullivan implies that $\Gamma(\mathrm{M})$ is an arithmetic group and the image of $\Gamma(\mathrm{M})$ has a finite index in the groups $\mathbb{S p}\left(2 b_{n}, \mathbb{Z}\right), \mathbb{S O}(2 p, q ; \mathbb{Z})$ and $\operatorname{ker}(\phi)$ is a finite group. A theorem of Borel implies that we can always find a subgroup of finite index $\Gamma$ in $\Gamma(\mathrm{M})$ such that $\Gamma$ acts freely on $\mathbb{S p}\left(2 b_{n}, \mathbb{R}\right) / \mathbb{U}\left(b_{n}\right)$ or on $\mathbb{S O}_{0}(2 p, q ; \mathbb{R}) / \mathbb{S O}(2 p) \times \mathbb{S O}(q)$. We will prove that $\Gamma$ acts without fixed points on $\mathcal{T}(\mathrm{M})$.

Let $\mathcal{K}$ be the Kuranishi space of the deformations of M. Suppose that there exists an element $g \in \Gamma$, such that $g(\tau)=\tau$ for some $\tau \in \mathcal{K} \subset \mathcal{T}(\mathrm{M})$.

From the local Torelli theorem we deduce that we may assume that the Kuranishi space $\mathcal{K}$ is embedded in $\mathcal{G}$, the classifying space of the Hodge structures of weight $\mathrm{n}$ on $H^{n}(\mathrm{M}, \mathbb{Z}) \otimes \mathbb{C}$. Griffiths proved in [14] that $\mathcal{G} \approx G / K$ where $\mathrm{G}$ in the odd dimensional case is $\mathbb{S p}\left(2 b_{n}, \mathbb{R}\right)$ and $\mathbb{S O}_{0}(2 p, q ; \mathbb{R})$ in the even dimensional and $K$ is a compact subgroup of $G$.

Let $K_{0}$ be the maximal compact subgroup of $G$. So we have a natural $C^{\infty}$ fibration

$$
K_{0} / K \subset G / K \rightarrow G / K_{0}
$$

Griffith's transversality theorem implies that $\mathcal{K}$ is transversal to the fibres $K_{0} / K$ of the fibration $G / K \rightarrow G / K_{0}$.

The first part of our theorem follows from the fact that $\mathcal{K}$ is transversal to the fibres $K_{0} / K$ of the fibration $G / K \rightarrow G / K_{0}$ and the following observation; if $g \in \Gamma$ fixes a point $\tau \in G / K_{0}$, then $g \in K_{0} \cap \Gamma{ }^{1}$ On the other hand

\footnotetext{
${ }^{1}$ We suppose that $K$ or $K_{0}$ acts on the right on $G$ and $\Gamma$ acts on the left on $G$.
} 
side it is easy to see that the local Torelli theorem implies that the action of $\Gamma$ on $\mathcal{K}$ is induced from the action $\Gamma$ on $G / K$ by left multiplications. So we can conclude that the action of $\Gamma$ preserves the fibration

$$
K_{0} / K \subset G / K \rightarrow G / K_{0} .
$$

The first part of our theorem follows directly from here and the fact that $\Gamma$ acts without fixed points on $G / K_{0}$.

The second part of the theorem, namely that the space $\Gamma \backslash \mathcal{T}(\mathrm{M})$ is a quasi projective follows directly from the fact that $\Gamma \backslash \mathcal{T}(\mathrm{M}) \rightarrow \Gamma(\mathrm{M}) \backslash \mathcal{T}(\mathrm{M})$ is a finite map and that $\Gamma(\mathrm{M}) \backslash \mathcal{T}(\mathrm{M})$ is a quasi projective variety according to [50]. Our theorem is proved.

\subsection{Construction of the Moduli Space of CY Manifolds}

According to Viehweg, the coarse moduli space of polarized CY manifolds

$$
\mathfrak{M}_{L}(\mathrm{M})=\mathcal{H}_{L} / \mathbb{S L}_{N+1}(\mathbb{C})
$$

is a quasi-projective variety. See [50]. On the other hand it is a standard that the coarse moduli space is just the following quotient:

$$
\mathfrak{M}_{L}(\mathrm{M})=\mathcal{T}_{L}(\mathrm{M}) / \Gamma_{L} .
$$

Theorem 33 There exists a finite cover $\mathcal{M}_{L}(M)$ of $\mathfrak{M}_{L}(M)=\mathcal{T}_{L}(M) / \Gamma_{L}$ with the following properties:

A. $\mathcal{M}_{L}(M)$ is a smooth algebraic variety.

B. Over $\mathcal{M}_{L}(M)$ there exists a family

$$
\mathcal{N}_{L} \rightarrow \mathcal{M}_{L}(M)
$$

of polarized CY manifolds with the following property: Let

$$
\pi_{\mathcal{C}}: \mathcal{Y} \rightarrow \mathcal{C}
$$

be any complex analytic family of polarized $C Y$ manifolds with a class of polarization $L$ such that at least one of its fibres of the family (5.2) is isomorphic as a polarized variety to a fibre of the family (5.1). Then there exists a unique complex analytic map

$$
\rho: \mathcal{C} \rightarrow \mathcal{M}_{L}(M) .
$$

which induces a holomorphic map between the families (5.2) and (5.1). The map between the families is defined uniquely up to a biholomorphic map $\phi$ of $M$ which induces the identity map on $H_{n}(M, \mathbb{Z})$. 
Proof: Let

$$
\psi: \Gamma_{L} \rightarrow H^{n}(\mathrm{M}, \mathbb{Z})
$$

be the natural representation of the group $\Gamma_{L}$. This results of Sullivan imply that the image $\psi\left(\Gamma_{L}\right)$ is an arithmetic group. According to Theorem 31, there exists a subgroup $\Gamma_{L}^{\prime \prime}$ of finite index in $\psi\left(\Gamma_{L}\right)$ such that $\psi\left(\Gamma_{L}\right)$ acts freely on the Teichmüller space $\mathcal{T}_{L}(\mathrm{M})$. From here we deduce that the space $\Gamma_{L}^{\prime \prime} \backslash \mathcal{T}_{L}(\mathrm{M})$ is smooth. Let us define $\Gamma_{L}^{\prime}:=\psi^{-1}\left(\Gamma_{L}^{\prime \prime}\right)$. Clearly $\Gamma_{L}^{\prime}$ is a subgroup of finite index in $\Gamma_{L}$. The local Torelli theorem implies that $\mathcal{T}_{L}(\mathrm{M}) / \Gamma_{L}^{\prime}$ will be a non-singular variety. From the definition of $\psi$ given by (5.3) it follows that $\operatorname{ker} \psi=G$ acts trivially on $H^{n}(\mathrm{M}, \mathbb{Z})$. The local Torelli Theorem implies that $G$ acts trivially on the Teichmüller space $\mathcal{T}_{L}(\mathrm{M})$. The existence and the properties of the family

$$
\mathcal{N}_{L} \rightarrow \mathcal{M}_{L}(M)
$$

follows from the existence and the properties of the family $\mathcal{Z}_{L} \rightarrow \mathcal{T}_{L}(M)$ proved in Theorem 27.

\section{The Theory of Determinant Line Bundles}

\subsection{Geometric Data}

In order to construct the determinant line bundle, we need the following data:

1. A smooth fibration of manifolds $\pi: \mathcal{X} \rightarrow \mathcal{M}(\mathrm{M})$. In our case it will be the smooth fibration of the family of CY manifolds over the moduli space as defined in Theorem 31 . Let $n=\operatorname{dim}_{\mathbb{C}} \mathrm{M}$.

2. A metric along the fibres, that is, a metric $\mathrm{g}(\tau)$ on the relative tangent bundle $\mathcal{T}(\mathcal{X} / \mathcal{M}(\mathrm{M}))$. In this paper the metric will be the families of CY metrics $\mathrm{g}(\tau)$ such that the class of cohomology $[\operatorname{Im}(g(\tau))]=L$ is fixed.

From this data we will construct the determinant line bundle $\mathcal{L}$ over the moduli space of $\mathrm{CY}$ manifolds $\mathcal{M}(\mathrm{M})$. We will consider the relative $\bar{\partial}_{\mathcal{X} / \mathcal{M}(\mathrm{M})}$ complex:

$$
\begin{gathered}
0 \longrightarrow \operatorname{ker} \bar{\partial}_{\mathcal{X} / \mathcal{M}(\mathrm{M})} \longrightarrow C^{\infty}(\mathcal{X}) \stackrel{\bar{\partial}_{0, \mathcal{X} / \mathcal{M}(\mathrm{M})}}{\longrightarrow} \Omega_{\mathcal{X} / \mathcal{M}(\mathrm{M})}^{0,1} \stackrel{\bar{\partial}_{1, \mathcal{X} / \mathcal{M}(\mathrm{M})}}{\longrightarrow} \\
\stackrel{\bar{\partial}_{1, \mathcal{X} / \mathcal{M}(\mathrm{M})}}{\longrightarrow} \Omega_{\mathcal{X} / \mathcal{M}(\mathrm{M})}^{0, n-1} \stackrel{\bar{\partial}_{n-1, \mathcal{X} / \mathcal{M}(\mathrm{M})}}{\longrightarrow} \Omega_{\mathcal{X} / \mathcal{M}(\mathrm{M})}^{0, n} \longrightarrow 0
\end{gathered}
$$


For each $\tau \in \mathcal{M}(\mathrm{M})$ and $k$, we will define $D$ to be:

$$
D_{k}:=\bar{\partial}_{k, \mathcal{X} / \mathcal{M}(\mathrm{M})}+\left(\bar{\partial}_{k, \mathcal{X} / \mathcal{M}(\mathrm{M})}\right)^{*}
$$

and

$$
D_{k, \tau}:=\left.D_{k}\right|_{\mathrm{M}_{\tau}}=\bar{\partial}_{k, \tau}+\left(\bar{\partial}_{k, \tau}\right)^{*} .
$$

Definition 34 We will call the above complex the relative Dolbault complex.

Let us define

$$
\mathcal{H}_{\tau}^{k}:=L^{2}\left(\mathrm{M}_{\tau}, \Omega_{\tau}^{0, k}\right) .
$$

Furthermore, as $\tau$ varies over $\mathcal{M}(\mathrm{M})$, the spaces $\left(\mathcal{H}_{\tau}^{k}\right)$ fit together to form continuous Hilbert bundles $\mathcal{H}^{k}$ over $\mathcal{M}(\mathrm{M}) .^{2}$ Thus we can view $\bar{\partial}_{k, \mathcal{X} / \mathcal{M}(\mathrm{M})}$ as bundle maps:

$$
\bar{\partial}_{k, \mathcal{X} / \mathcal{M}(\mathrm{M})}: \mathcal{H}^{k} \rightarrow \mathcal{H}^{k+1} .
$$

The Hilbert bundles $\mathcal{H}^{k}$ carry $L^{2}$ metrics by definition.

Now we are ready to construct the Determinant line bundle $\mathcal{L}$ of the operator $\bar{\partial}_{\mathcal{X} / \mathcal{M}(\mathrm{M})}$. We will recall some basic consequences of the ellipticity of $D_{\tau}$. Each fibre $\mathcal{H}_{\tau}^{k}$ of the Hilbert bundles $\mathcal{H}^{k}$ decomposes into direct sums of eigen spaces of non-negative Laplacians $D_{k} D_{k}^{*}$ and $D_{k}^{*} D_{k}$. The spectrums of these operators are discrete, and the nonzero eigen values $\left\{\lambda_{k, i}\right\}$ of $D_{k}^{*} D_{k}$ and $D_{k} D_{k}^{*}$ agree and $\mathrm{D}_{k}$ defines a canonical isomorphism between the corresponding eigen spaces.

Definition 35 1. Let

$$
\mathcal{U}_{a}:\left\{\tau \in \mathcal{M}(M) \mid a \notin \operatorname{Spec}\left(D_{k} D_{k}^{*}\right)\right.
$$

for $0 \leq k \leq n$ and any $a>0\}$. ( $U_{a}$ are open sets in $\mathcal{M}(M)$ and they form an open covering of $\mathcal{M}(M)$ since the spectrum of $D_{\tau}^{*} D_{\tau}$ is discrete.)

2. Let the fibres of $\mathcal{H}_{a}^{k}$ be the vector subspaces $\mathcal{H}_{\tau, a}^{k}$ in $\mathcal{H}_{\tau}^{k}$ spanned by eigen vectors with eigen values less than a for $\tau \in \mathcal{U}_{a}$. Then we can define the complex:

$$
\begin{gathered}
0 \longrightarrow \Gamma\left(\mathcal{U}_{a}, \mathcal{O}_{\mathcal{U}_{a}}\right) \longrightarrow \mathcal{H}_{a}^{0} \stackrel{\bar{\partial}_{0, \mathcal{X} / \mathcal{M}(M)}}{\longrightarrow} \cdots \\
\ldots \longrightarrow \mathcal{H}_{a}^{n-1} \stackrel{\bar{\partial}_{n-1, \mathcal{X} / \mathcal{M}(M)}}{\longrightarrow} \mathcal{H}_{a}^{n} \longrightarrow \operatorname{ker}\left(D_{n-1} \circ D_{n}^{*}\right) \longrightarrow 0 .
\end{gathered}
$$

If $b>a$ we set $\mathcal{H}_{a, b}^{k}:=\mathcal{H}_{b}^{k} / \mathcal{H}_{a}^{k}$. The spaces $\mathcal{H}_{a}^{k}$ form smooth finite dimensional $C^{\infty}$ bundles over an open set $\mathcal{U}^{a} \subset \mathcal{M}(M)$. For the proof of this fact see [1].

\footnotetext{
${ }^{2}$ These bundles are not smooth since the composition $L^{2} \times C^{\infty} \rightarrow L^{2}$ is not a differentiable map.
} 
710 A. Todorov

6.2. Construction of the Generating Sections $\operatorname{det}\left(D_{a}\right)$ over $\mathcal{U}_{a}$ Definition 36 Let

$$
\omega_{1}^{k}, \ldots, \omega_{m_{k}}^{k}, \psi_{1}^{k}, \ldots, \psi_{N_{k}}^{k}, \phi_{1}^{k}, \ldots, \phi_{M_{k}}^{k}
$$

be an orthonormal basis in the trivial vector bundle $\mathcal{H}_{a}^{k}$ over $\mathcal{U}_{a}$, where

$$
D_{k} \omega_{i}^{k}=0, \bar{\partial}_{k}^{*}\left(\bar{\partial}_{k} \psi_{j}^{k}\right)=\lambda_{j}^{k} \psi_{j}^{k}, \bar{\partial}_{k}\left(\bar{\partial}_{k}^{*} \phi_{j}^{k}\right)=\lambda_{j}^{k} \phi_{j}^{k}, \phi_{j}^{k} \in \operatorname{Im} \bar{\partial}_{k-1, \mathcal{X} / \mathcal{M}(M)}
$$

and

$$
\psi_{j}^{k} \in \operatorname{Im}\left(\bar{\partial}_{k, \mathcal{X} / \mathcal{M}(M)}^{*}\right)
$$

for $1 \leq i \leq k$ and $0<\lambda_{j}<a$ for $1 \leq j \leq N$. Let

$$
\begin{aligned}
\operatorname{det}\left(\bar{\partial}_{k, a}\right)= & \left(\omega_{1}^{k} \wedge \cdots \wedge \omega_{m_{k}}^{k} \wedge\left(\bar{\partial}_{k-1, \mathcal{X} / \mathcal{M}(M)} \psi_{1}^{k-1}\right)\right. \\
& \left.\wedge \cdots \wedge\left(\bar{\partial}_{k-1, \mathcal{X} / \mathcal{M}(M)} \psi_{N_{k}}^{k-1}\right) \wedge \phi_{1}^{k} \wedge \cdots \wedge \phi_{M_{k}}^{k}\right)^{(-1)^{k}} .
\end{aligned}
$$

We will define the line bundle $\mathcal{L}$ restricted on $\mathcal{U}_{a}$ as follows:

$$
\mathcal{L}^{a}:=\left.\mathcal{L}\right|_{\mathcal{U}_{a}}=\otimes_{k=0}^{n}\left(\bigwedge^{\operatorname{dim} \mathcal{H}_{a}^{k}} \mathcal{H}_{a}^{k}\right)^{(-1)^{k}}
$$

Definition 37 The generator $\operatorname{det}\left(\bar{\partial}_{a}\right)$ of $\mathcal{L}^{a}:=\left.\mathcal{L}\right|_{\mathcal{U}^{a}}$ is defined as follows:

$$
\operatorname{det}\left(\bar{\partial}_{a}\right):=\underset{k}{\otimes} \operatorname{det}\left(\bar{\partial}_{k, a}\right) \text {. }
$$

We will define how we patch together $\mathcal{L}^{a}$ and $\mathcal{L}^{b}$ over $\mathcal{U}^{a} \cap \mathcal{U}^{b}{ }^{3}$ On that intersection we have:

$$
\mathcal{L}^{b}=\mathcal{L}^{a} \otimes \mathcal{L}^{a, b}
$$

where

$$
\mathcal{L}^{a, b}:=\otimes_{k}\left(\operatorname{det} \mathcal{H}_{a, b}^{k}\right)^{(-1)^{k}}
$$

on $\mathcal{U}^{a} \cap \mathcal{U}^{b}$. We can identify $\mathcal{L}^{a}, b$ over $\mathcal{U}^{a} \cap \mathcal{U}^{b}$ with the line bundle spanned by the section

$$
\operatorname{det}\left(\bar{\partial}_{a, b}\right)=\otimes_{k=0}^{n} \operatorname{det}\left(\bar{\partial}_{k, a, b}\right)^{(-1)^{k}}
$$

where

$$
\begin{aligned}
\operatorname{det}\left(\bar{\partial}_{k, a, b}\right):= & \left(\left(\bar{\partial}_{k-1, \mathcal{X} / \mathcal{M}(\mathrm{M})} \psi_{1}^{k-1}\right)\right. \\
& \left.\wedge \cdots \wedge\left(\bar{\partial}_{k-1, \mathcal{X} / \mathcal{M}(\mathrm{M})} \psi_{N_{k}}^{k-1}\right) \wedge \phi_{1}^{k} \wedge \cdots \wedge \phi_{M_{k}}^{k}\right),
\end{aligned}
$$

$\phi_{j}^{k} \in \operatorname{Im} \bar{\partial}_{k-1, \mathcal{X} / \mathcal{M}(\mathrm{M})}, \psi_{j}^{k} \in \operatorname{Im}\left(\bar{\partial}_{0, \mathcal{X} / \mathcal{M}(\mathrm{M})}^{*}\right), \Delta_{k} \phi_{j}^{k}=\lambda_{j}^{k} \phi_{j}^{k}, \Delta_{k}\left(\bar{\partial}\left(\psi_{i}^{k-1}\right)\right)=$ $\lambda_{i}^{k}\left(\bar{\partial}\left(\psi_{i}^{k-1}\right)\right)$ and $a<\lambda_{i}^{k}<b$.

${ }^{3}$ We may suppose that $b>a$. 
Remark 38 We can view $\operatorname{det}\left(\bar{\partial}_{a, b}\right)$ as a section of the line bundle $\mathcal{L}^{a, b}$ over $\mathcal{U}^{a} \cap \mathcal{U}^{b}$. It defines canonical smooth isomorphisms over $\mathcal{U}^{a} \cap \mathcal{U}^{b}$ :

$$
\mathcal{L}^{a} \rightarrow \mathcal{L}^{a} \otimes \mathcal{L}^{a, b}=\mathcal{L}^{b}\left(s \rightarrow s \otimes \operatorname{det}\left(\bar{\partial}_{a, b}\right)\right.
$$

for all $0<a<b$.

We define the determinant line bundle $\mathcal{L}$ by patching the trivial line bundles $\mathcal{L}^{a}$ over $\mathcal{U}^{a}$ by using the canonical isomorphism defined in Remark 38 .

\subsection{The Description of the Quillen Metric on $\mathcal{L}$}

We now proceed to describe the Quillen metric on $\mathcal{L}$. Let us fix $a>0$. Then the subbundles $\mathcal{H}_{a}^{k}$ of the Hilbert bundles $\mathcal{H}^{k}$ on $\mathcal{U}_{a}$ inherit metrics from $\mathcal{H}^{k}$. According to standard facts from linear algebra, metrics are induced on determinants, duals, and tensor products. So the $\mathcal{L}^{a}$ inherits a natural metric. We will denote by $\mathrm{g}^{a}$ the $L^{2}$ norm of the section $\operatorname{det}\left(\bar{\partial}_{a}\right)$. Clearly,

$$
g^{a}=\prod_{k=0}^{n}\left(\lambda_{1}^{k} \ldots \lambda_{n_{k}}^{k}\right)^{(-1)^{k}},
$$

and $\lambda_{i}^{k}$ are all nonzero eigen values of the operators $\bar{\partial}_{k}^{*} \bar{\partial}_{k-1}$ which are less than $a$.

If $b>a$, then under the isomorphism defined in Remark 38, we have two metrics on $\mathcal{L}^{b}$ and their ratio is a real number equal to the $L^{2}$ norm of the section $\left\|\operatorname{det}\left(\bar{\partial}_{a, b}\right)\right\|^{2}$. The definition of the section $\operatorname{det}\left(\bar{\partial}_{a, b}\right)$ implies that we have the following formula:

$$
\begin{gathered}
\left\|\operatorname{det}\left(\bar{\partial}_{a, b}\right)\right\|^{2}=\prod_{k=0}^{n} \prod_{i=1}\left\|\phi_{i}^{k}\right\|^{2} \prod_{j=1}\left\|\bar{\partial} \psi_{j}^{k}\right\|^{2(-1)^{k}}= \\
=\prod_{i=1}\left\|\phi_{i}^{k}\right\|^{2} \prod_{j=1}\left\langle\bar{\partial}_{k}^{*} \bar{\partial}_{k-1} \psi_{j}^{k}, \psi_{j}^{k}\right\rangle^{(-1)^{k}}=\prod\left(\lambda_{i}^{k}\right)^{(-1)^{k}}
\end{gathered}
$$

where $\lambda_{i}^{k}$ are all the non-zero eigen values of the operators $\bar{\partial}_{k}^{*} \bar{\partial}_{k-1}$ such that $a<\lambda_{i}^{k}<b$. In other words, on $\mathcal{U}^{a} \cap \mathcal{U}^{b}$

$$
g^{b}=g^{a} \prod_{a<\lambda_{i}^{k}<b}\left(\lambda_{i}^{k}\right)^{(-1)^{k}} .
$$

To correct this discrepancy, we define

$$
\bar{g}^{a}=\left.g^{a} \operatorname{det}\left(\bar{\partial}^{*} \bar{\partial}\right)\right|_{\lambda>a},
$$


712 A. TODOROV

where

$$
\begin{aligned}
\left.\operatorname{det}\left(\bar{\partial}^{*} \bar{\partial}\right)\right|_{\lambda>a} & =\prod_{k=1}^{n} \operatorname{det}\left(\left.\bar{\partial}_{k}^{*} \bar{\partial}_{k-1}\right|_{\lambda>a}\right)^{(-1)^{k}} \\
\operatorname{det}\left(\left.\bar{\partial}_{k}^{*} \bar{\partial}_{k-1}\right|_{\lambda>a}\right) & =-\exp \left(-\left(\zeta_{k}^{a}\right)^{\prime}(0)\right),
\end{aligned}
$$

and

$$
\zeta_{k}^{a}(s)=\sum_{\lambda_{i}>a}^{\infty}\left(\lambda_{i}^{k}\right)^{s} .
$$

The crucial property of this regularization is that it behaves properly with respect to the finite number of eigen values, i.e.

$$
\operatorname{det}\left(\left.\bar{\partial}_{k}^{*} \bar{\partial}_{k-1}\right|_{\lambda>b}\right)=\operatorname{det}\left(\left.\bar{\partial}_{k}^{*} \bar{\partial}_{k-1}\right|_{\lambda>a}\right) \prod_{a<i<b}^{N} \lambda_{i}^{k}
$$

on the intersection $\mathcal{U}^{a} \cap \mathcal{U}^{b}$. From the last remark we deduce that $\bar{g}^{a}$ and $\bar{g}^{b}$ agree on $\mathcal{U}^{a} \cap \mathcal{U}^{b}$. Thus $\bar{g}^{a}$ and $\bar{g}^{b}$ patch together to a Hermitian metric $\mathrm{g}^{\mathcal{L}}$ on $\mathcal{L}$. The metric $\mathrm{g}^{\mathcal{L}}$ will be called the Quillen metric on $\mathcal{L}$.

Definition 39 We will define the holomorphic Ray Singer analytic torsion $I(M)$ for the odd dimensional $C Y$ manifold $M$ as follows:

$$
I(M):=\frac{1}{2} \log \left(\prod_{q=1}^{n}\left(\operatorname{det}\left(\triangle_{q}^{\prime}\right)^{(-1)^{q}}\right) .\right.
$$

See [34].

Remark 40 It is easy to see that if $M$ is a $C Y$ manifold and $\operatorname{dim}_{\mathbb{C}} M=2 n$, then $\log I(M)=0$. We know from the results in [47] that for odd dimensional $C Y$ manifolds $\exp (I(M))>0$. So from now on we will consider only odd dimensional CY manifolds.

We will need the following result from [6] on p. 55:

Theorem 41 The Quillen norm of the $C^{\infty}$ section $\operatorname{det}\left(\bar{\partial}_{a}\right)$ on $\mathcal{U}_{a}$ of $\mathcal{L}$ is equal to $\exp (-I(M))$.

Proof: It follows from Definition 36 of the section $\left.\operatorname{det}(\bar{\partial})\right|_{\mathcal{U}^{a}}$ of $\mathcal{L}$ and the definition of the Quillen metric that at each point $\tau \in \mathcal{M}(\mathrm{M})$ the following formula is true:

$$
\left\|\operatorname{det}(\bar{\partial})_{\tau}\left|\mathcal{U}^{a} \|_{Q}^{2}=\exp \left(\mathrm{I}\left(\mathrm{M}_{\tau}\right)\right)\right|_{\mathcal{U}^{a}}\right.
$$

where $\left\|\operatorname{det}(\bar{\partial})_{\tau} \mid \mathcal{U}^{a}\right\|_{Q}^{2}$ means the Quillen norm of the $\operatorname{section} \operatorname{det}(\bar{\partial})_{\tau}{\mid \mathcal{U}^{a}}$. Theorem 41 is proved. 


\section{Construction of a $C^{\infty}$ Non-Vanishing Section of the Determinant Line Bundle $\mathcal{L}$ for Odd Dimensional CY Manifolds}

\subsection{Some Preliminary Results}

Let us denote by

$$
\pi_{*}\left(\omega_{\mathcal{X} / \mathcal{M}(\mathrm{M})}\right):=\pi_{*}\left(\Omega_{\mathcal{X} / \mathcal{M}(\mathrm{M})}^{n, 0}\right)
$$

the relative dualizing sheaf. The local sections of $\pi_{*}\left(\omega_{\mathcal{X} / \mathcal{M}(\mathrm{M})}\right)$ are families of holomorphic $n$-forms $\omega_{\tau}$ on $\mathrm{M}_{\tau}$. Then on $\pi_{*}\left(\omega_{\mathcal{X} / \mathcal{M}(\mathrm{M})}\right)$ we have a natural $L^{2}$ metric defined as follows:

$$
\left\|\omega_{\tau}\right\|^{2}:=(-1)^{\frac{n(n-1)}{2}}(\sqrt{-1})^{n} \int_{\mathrm{M}} \omega_{\tau} \wedge \overline{\omega_{\tau}}
$$

Theorem 42 If the dimension of the $C Y$ manifold is even, then $\mathcal{L}$ is isomorphic to the dual of the line bundle $\pi_{*}\left(\omega_{\mathcal{X} / \mathcal{M}(M)}\right)$. If the dimension of the $C Y$ manifold is odd, then $\mathcal{L}$ is isomorphic to the line bundle $\pi_{*}\left(\omega_{\mathcal{X} / \mathcal{M}(M)}\right)$ over $\mathcal{M}(M)$.

Proof: The definition of CY manifold states that:

$$
\operatorname{dim}_{\mathbb{C}} H^{j}\left(\mathrm{M}, \mathcal{O}_{\mathrm{M}}\right)= \begin{cases}1 & j=0 \text { or } j=n \\ 0 & \text { for } j \neq 0 \text { or } n\end{cases}
$$

Formula (7.2) and the definition of CY manifolds imply that

$$
R^{q} \pi_{*} \mathcal{O}_{\mathrm{M}}= \begin{cases}\left(\pi_{*} \omega_{\mathcal{X} / \mathcal{M}(\mathrm{M})}\right)^{*} & \text { for } j=n \\ \mathcal{O}_{\mathcal{M}(\mathrm{M})} & \text { for } j \neq n\end{cases}
$$

From the definition of $\mathcal{L}$ it follows that

$$
\mathcal{L} \backsim \prod_{q=0}^{n}(-1)^{q} \operatorname{det}\left(R^{q} \pi_{*} \mathcal{O}_{\mathrm{M}}\right)
$$

Combining (7.2) and (7.3), we directly deduce Theorem 42.

Corollary 43 Let $M$ be a $C Y$ manifold of odd dimension $n=2 m+1$. Then the index of the operator $\bar{\partial}$ on the complex defined in Definition 34 is zero. 


\subsection{Holomorphic Structure on the Determinant line Bundle $\mathcal{L}$}

In [6] a canonical smooth isomorphism is constructed between the holomorphic determinant of the Grothendieck-Knudsen-Mumford and Quillen determinant bundle. More precisely, the following theorem is proved:

Theorem 44 Let

$$
\pi: \mathcal{X} \rightarrow \mathcal{M}(M)
$$

be a holomorphic fibration with smooth fibres. Suppose $\mathcal{X}$ admits a closed $(1,1)$ form $\psi$ which restricts to a Kähler metric on each fibre. Let $\mathcal{E} \rightarrow \mathcal{X}$ be a holomorphic Hermitian bundle with its Hermitian connection. Then the determinant line bundle $\mathcal{L} \rightarrow \mathcal{M}(M)$ of the relative $\bar{\partial}$ complex (coupled to $\mathcal{E}$ ) admits a holomorphic structure. The canonical connection (constructed in [5]) on $\mathcal{L}$ is the Hermitian connection for the Quillen metric. Finally, if the index of $\bar{\partial}$ is zero, the section $\operatorname{det}\left(\bar{\partial}_{E}\right)$ of $\mathcal{L}$ is holomorphic.

From now on we will consider the family of $\mathrm{CY}$ manifolds $\mathcal{X} \rightarrow \mathcal{M}(\mathrm{M})$ as defined in Theorem 33 together with the trivial line bundle $\mathcal{E}$ over $\mathcal{M}(\mathrm{M})$. It is easy to see that the family $\mathcal{X} \rightarrow \mathcal{M}(\mathrm{M})$ fulfills the conditions of Theorem 44 .

\subsection{Construction of a $C^{\infty}$ Section of the Determinant Line Bundle $\mathcal{L}$ with Quillen Norm Equal to Ray-Singer Analytic Torsion}

Definition 45 Let

$$
\mathcal{H}_{+}=\underset{k}{\oplus} L^{2}\left(M, \Omega_{M}^{0,2 k}\right), \quad \mathcal{H}_{-}=\underset{k}{\oplus} L^{2}\left(M, \Omega_{M}^{0,2 k+1}\right)
$$

and

$$
D=\bar{\partial}_{\mathcal{X} / \mathcal{M}(M)}+\bar{\partial}_{\mathcal{X} / \mathcal{M}(M)}^{*}
$$

Theorem 46 Let $M$ be a $C Y$ manifold of odd dimension, then as a $C^{\infty}$ bundle the determinant line bundle $\mathcal{L}$ is trivial and, moreover, there exists a global $C^{\infty}$ section $\operatorname{det}(\bar{\partial})$ of

$$
\mathcal{L} \rightarrow \mathcal{M}(M)
$$

which has no zeroes on $\mathcal{M}(M)$ and whose Quillen norm is the exponential of the Ray Singer Analytic Torsion. 
Proof: The proof of Theorem 46 is based on the following three Theorems:

Theorem 47 The Chern form of the relative dualizing sheaf $\pi_{*} \omega_{\mathcal{X} / \mathcal{M}(M)}$ is given locally by the formula

$$
c_{1}\left(\pi_{*} \omega_{\mathcal{X} / \mathcal{M}(M)}\right)=d d^{c}\left(\left\langle\omega_{\tau}, \omega_{\tau}\right\rangle\right)=-\operatorname{Im}(\text { Weil-Petersson metric }),
$$

where $\omega_{\tau}$ is a holomorphic family of holomorphic n forms. (See [45].)

Theorem 48 The following formula holds true locally on the moduli space $\mathcal{M}(M)$ :

$$
d d^{c}\left(\log \left(\operatorname{det}\left(\triangle_{0}\right)\right)\right)=\operatorname{Im}(\text { Weil-Petersson metric }) .
$$

(See $[47])$.

Theorem 49 (i) $I(M)=-\log \operatorname{det}\left(\triangle_{0}\right)$. (ii) Around each point $\tau \in \mathcal{U}_{\tau} \subset$ $\mathcal{M}(M)$ we have

$$
\left.\exp (I(M))\right|_{\mathcal{U}_{\tau}}=<\omega, \omega>|\phi|^{2},
$$

where $\phi$ is a holomorphic function on $\mathcal{U}$. And (iii) the positive function $\operatorname{det}\left(\triangle_{0}\right)$ is bounded by a constant $C$ if the dimension of $M$ is three, i.e.

$$
\operatorname{det}\left(\triangle_{0}\right)<C
$$

(See [47]). We will prove the following Lemma:

Lemma 50 The first Chern class $c_{1}(\mathcal{L})$ of the $C^{\infty}$ determinant line bundle $\mathcal{L}$ is equal to zero in $H^{2}(\mathcal{M}(M), \mathbb{Z})$.

Proof: Theorem 42 implies that when the CY manifold $\mathrm{M}$ has an odd dimension, then the determinant line bundle $\mathcal{L}$ is isomorphic to the relative dualizing sheaf $\pi_{*}\left(\omega_{\mathcal{X} / \mathcal{M}(\mathrm{M})}\right)$. So we need to prove that

$$
c_{1}\left(\pi_{*}\left(\omega_{\mathcal{X} / \mathcal{M}(\mathrm{M})}\right)\right)=0 .
$$

The proof of Lemma 50 is based on the following observation: Notice that the definition of the exponential of the Ray Singer analytic torsion implies that $\exp \left(\mathrm{I}_{\tau}\left(\mathrm{M}_{\tau}\right)\right)$ is a positive function different from a constant on $\mathcal{M}(\mathrm{M})$. From Theorem 49 we know that we have the following local expression of $\exp \left(\mathrm{I}\left(\mathrm{M}_{\tau}\right)\right)$ :

$$
\left.\exp \left(\mathrm{I}\left(\mathrm{M}_{\tau}\right)\right)\right|_{\mathcal{U}}=\left\|\omega_{\tau}\right\|^{2}|\phi|^{2}
$$

where $\omega_{\tau}$ is a holomorphic family of holomorphic n-forms on $M_{\tau}, \phi$ is a holomorphic function on $\mathcal{U}$ and $\left\|\omega_{\tau}\right\|^{2}$ is defined by (7.1). Theorems 48 and 47 imply that

$$
\frac{\sqrt[2]{-1}}{2}\left(\partial \bar{\partial}\left(\mathrm{I}\left(\mathrm{M}_{\tau}\right)\right)\right)=c_{1}(\mathcal{L})=c_{1}\left(\pi_{*}\left(\omega_{\mathcal{X} / \mathcal{M}(\mathrm{M})}\right)\right) .
$$


Since

$$
\left.\partial \bar{\partial}\left(\mathrm{I}_{\tau}\right)\right)=d\left(\bar{\partial}\left(\mathrm{I}\left(\mathrm{M}_{\tau}\right)\right)\right)
$$

we deduce that

$$
\left.\frac{\sqrt[2]{-1}}{2} \partial \bar{\partial}\left(\mathrm{I}\left(\mathrm{M}_{\tau}\right)\right)=c_{1}(\mathcal{L})=\frac{\sqrt[2]{-1}}{2} d\left(\bar{\partial}\left(\mathrm{I}_{(} \mathrm{M}_{\tau}\right)\right)\right)=d \alpha
$$

So $c_{1}(\mathcal{L})=0$ in $H^{2}(\mathcal{M}(\mathrm{M}), \mathbb{Z})$. This proves Lemma 50 .

Corollary 51 The determinant line bundle $\mathcal{L}$ is trivial as a $C^{\infty}$ bundle.

So the first part of Theorem 46 is proved. The proof of the second part of Theorem 46 is based on the following Lemma:

Lemma 52 There exists a nonvanishing global section $\operatorname{det}(\bar{\partial})$ of the determinant line bundle $\mathcal{L}$ such that the Quillen norm of $\operatorname{det}(\bar{\partial})$ is $\exp (I(M))$.

Proof: From Corollary 51 we can conclude the existence of a global $C^{\infty}$ section $\omega_{\tau}$ of the line bundle

$$
\mathcal{L} \rightarrow \mathcal{M}(\mathrm{M})
$$

which has no zeroes on $\mathcal{M}(\mathrm{M})$ and which for each $\tau \in \mathcal{M}(\mathrm{M})$ has $L^{2}$ norm 1 , i.e. we have $\left\|\omega_{\tau}\right\|^{2}=1$. Since $M_{\tau}$ is an odd dimensional CY manifold, we know from Theorem 42 that $\mathcal{L}$ is isomorphic to $\pi_{*}\left(\omega_{\mathcal{X} / \mathcal{M}(\mathrm{M})}\right)$. The nonvanishing section $\omega_{\tau}$ of the determinant line bundle $\mathcal{L}$ can be interpreted as a family of $(2 \mathrm{n}+1,0)$ forms $\omega_{\tau}$ which generate the kernel of

$$
D^{*}: \mathcal{H}_{-} \rightarrow \mathcal{H}_{+} .
$$

The kernel of $D: \mathcal{H}_{+} \rightarrow \mathcal{H}_{-}$is generated by the constant 1 . This follows directly from the definition of the CY manifold.

From Definition 36 of the section $\operatorname{det}\left(\bar{\partial}_{a}\right)$ on the open set $\mathcal{U}_{a}$ in $\mathcal{M}(\mathrm{M})$, as well as the existence of a $C^{\infty}$ family of antiholomorphic forms $\omega_{\tau}$ with $L^{2}$ norm 1 , which trivializes $R^{2 n+1} \pi_{*}\left(\mathcal{O}_{\mathcal{X}}\right)$ over $\mathcal{M}(\mathrm{M})$, and the definition of the transition functions $\left\{\sigma_{a, b}\right\}$ of $\mathcal{L}$ with respect to the covering $\left\{\mathcal{U}_{a}\right\}$, we deduce that for $b>a$ we have on $\mathcal{U}_{a} \cap \mathcal{U}_{b}$

$$
\operatorname{det}\left(\bar{\partial}_{b}\right)=\operatorname{det}\left(\bar{\partial}_{b}\right)\left(\sigma_{a, b}\right) .
$$

This fact and Theorem 41 imply that we have constructed a global $C^{\infty}$ section $\operatorname{det}(\bar{\partial})$ of $\mathcal{L}$ whose Quillen norm is $\exp (-\mathrm{I}(\mathrm{M}))$. So the determinant line bundle $\mathcal{L}$ is a trivial $C^{\infty}$ line bundle. Theorem 46 is proved.

Corollary 53 The determinant line bundle as a holomorphic bundle is flat over $\mathcal{M}(M)$. 


\section{The Discriminant Locus and the analogue of the Dedekind Eta Function for odd-dimensional Calabi- Yau Manifolds}

\subsection{Metrics on Vector Bundles with Logarithmic Growth}

In Theorem 31 we constructed the moduli space $\mathcal{M}(\mathrm{M})$ of $\mathrm{CY}$ manifolds. From the results in [50] and Theorem 31 we know that $\mathcal{M}(\mathrm{M})$ is a quasiprojective non-singular variety. Using Hironaka's resolution theorem, we may suppose that

$$
\mathcal{M}(\mathrm{M}) \subset \overline{\mathcal{M}(\mathrm{M})}
$$

where

$$
\overline{\mathcal{M}(\mathrm{M})} \ominus \mathcal{M}(\mathrm{M})=\mathfrak{D}
$$

is a divisor with normal crossings. We need now to show how we will extend the determinant line bundle $\mathcal{L}$ to a line bundle $\overline{\mathcal{L}}$ to $\overline{\mathcal{M}(\mathrm{M})}$. For this reason we are going to recall the following definitions and results from [30]. We will look at policylinders

$$
\mathrm{D}^{N} \subset \overline{\mathcal{M}(\mathrm{M})}
$$

where $\mathrm{D}$ is the unit disk,

$$
N=\operatorname{dim} \overline{\mathcal{M}(\mathrm{M})}
$$

such that

$$
\mathrm{D}^{N} \cap \mathfrak{D}_{\infty}=\left\{\text { union of hyperplanes; } \tau_{1}=0, \ldots, \tau_{k}=0\right\} .
$$

Hence,

$$
\mathrm{D}^{N} \cap \mathcal{M}(\mathrm{M})=\left(\mathrm{D}^{*}\right)^{k} \times \mathrm{D}^{N-k} .
$$

On $\mathrm{D}^{*}$ we have the Poincaré metric

$$
d s^{2}=\frac{|d z|^{2}}{|z|^{2}(\log |z|)^{2}}
$$

and on $\mathrm{D}$ we have the simple metric $|d z|^{2}$, giving us a product metric on $\left(\mathrm{D}^{*}\right)^{k} \times \mathrm{D}^{N-k}$ which we call $\omega^{(P)}$.

A complex-valued $C^{\infty}$ p-form $\eta$ on $\mathcal{M}(\mathrm{M})$ is said to have Poincaré growth on $\overline{\mathcal{M}(\mathrm{M})} \ominus \mathcal{M}(\mathrm{M})$ if there is a set of if policylinders

$$
\mathcal{U}_{\alpha} \subset \overline{\mathcal{M}(\mathrm{M})}
$$

covering

$$
\overline{\mathcal{M}(\mathrm{M})} \ominus \mathcal{M}(\mathrm{M})
$$


such that in each $\mathcal{U}_{\alpha}$ an estimate of the following type holds:

$$
\mid \eta\left(\tau_{1}, \ldots, \tau_{N} \mid \leq C_{\alpha} \omega_{\mathcal{U}_{\alpha}}^{(P)}\left(\tau_{1}, \overline{\tau_{1}}\right) \ldots C_{\alpha} \omega_{\mathcal{U}_{\alpha}}^{(P)}\left(\tau_{N}, \overline{\tau_{N}}\right) .\right.
$$

This property is independent of the covering $\mathcal{U}_{\alpha}$ of $\overline{\mathcal{M}(\mathrm{M})} \ominus \mathcal{M}(\mathrm{M})$ but depends on the compactification $\overline{\mathcal{M}(\mathrm{M})}$. If $\eta_{1}$ and $\eta_{2}$ both have Poincaré growth on

$$
\overline{\mathcal{M}(\mathrm{M})} \ominus \mathcal{M}(\mathrm{M})
$$

then so does $\eta_{1} \wedge \eta_{2}$. The basic property of the Poincaré growth is the following:

Theorem 54 A p-form $\eta$ with a Poincaré growth on $\overline{\mathcal{M}(M)} \ominus \mathcal{M}(M)=\mathfrak{D}$ has the property that for every $C^{\infty}(r-p)$ form $\psi$ on $\overline{\mathcal{M}(M)}$ we have:

$$
\int_{\overline{\mathcal{M}(M)} \ominus \mathcal{M}(M)}|\eta \wedge \psi|<\infty .
$$

Hence, $\eta$ defines a current $[\eta]$ on $\overline{\mathcal{M}(M)}$.

Proof: For the proof see [30].

A complex valued $C^{\infty}$ p-form $\eta$ on $\overline{\mathcal{M}(\mathrm{M})}$ is good on $M$ if both $\eta$ and $d \eta$ have Poincaré growth. Let $\mathcal{E}$ be a vector bundle on $\mathcal{M}(\mathrm{M})$ with a Hermitian metric $h$. We will call $\mathrm{h}$ a good metric on $\overline{\mathcal{M}(\mathrm{M})}$ if the following holds:

1. If for all $\mathrm{x} \in \overline{\mathcal{M}(\mathrm{M})} \ominus \mathcal{M}(\mathrm{M})$ there exist sections $e_{1}, \ldots, e_{m}$ of $\mathcal{E}$ which form a basis of $\left.\mathcal{E}\right|_{D^{r} \ominus\left(D^{r} \cap \mathfrak{D}_{\infty}\right)}$.

2. In a neighborhood $D^{r}$ of $\mathrm{x}$ in which $\overline{\mathcal{M}(\mathrm{M})} \ominus \mathcal{M}(\mathrm{M})$ is given by

$$
z_{1} \times \cdots \times z_{k}=0 .
$$

3. The metric $\mathrm{h}_{i \bar{j}}=\mathrm{h}\left(e_{i}, e_{j}\right)$ has the following properties:

$$
\text { (a) } \quad\left|h_{i \bar{j}}\right| \leq C\left(\sum_{i=1}^{k} \log \left|z_{i}\right|\right)^{2 m},(\operatorname{det}(h))^{-1} \leq C\left(\sum_{i=1}^{k} \log \left|z_{i}\right|\right)^{2 m}
$$

for some $C>0, m \geq 0$. (b) The 1 -forms $\left((d h) h^{-1}\right)_{i \bar{j}}$ are good forms on $\overline{\mathcal{M}(\mathrm{M})} \cap D^{N}$.

It is easy to prove that there exists a unique extension $\overline{\mathcal{E}}$ of $\mathcal{E}$ on $\overline{\mathcal{M}(\mathrm{M})}$, i.e. $\overline{\mathcal{E}}$ is defined locally as holomorphic sections of $\mathcal{E}$ which have a finite norm in $h$.

Theorem 55 Let $(\mathcal{E}, h)$ be a vector bundle with a good metric on $\mathcal{M}(M)$, then the Chern classes $c_{k}(\mathcal{E}, h)$ are good forms on $\overline{\mathcal{M}(M)}$ and the currents $\left[c_{k}(\mathcal{E}, h)\right]$ represent the cohomology classes

$$
c_{k}(\mathcal{E}, h) \in H^{2 k}(\overline{\mathcal{M}(M)}, \mathbb{Z}) .
$$

Proof: For the proof see [30]. 


\subsection{Applications of Mumford's Results to the Moduli of CY}

We are going to prove the following result:

Theorem 56 Let

$$
\pi: \mathcal{X} \rightarrow \mathcal{M}(M)
$$

be the flat family of non-singular CY manifolds. Let $\pi_{*}\left(\Omega_{\mathcal{X} / \mathcal{M}(M)}^{n, 0}\right)$ be equipped with the metric $h$ defined in (7.1). Then $h$ is a good metric.

Proof: Let $\tau_{0} \in \mathfrak{D}=\overline{\mathcal{M}(\mathrm{M})} \ominus \mathcal{M}(\mathrm{M})$. Let $\mathrm{D}$ be a one dimensional disk in $\overline{\mathcal{M}(\mathrm{M})}$ which intersects $\mathfrak{D}$ in $\tau_{0}$ and

$$
\mathrm{D}^{*}=\mathrm{D} \ominus \tau_{0} \subset \mathcal{M}(\mathrm{M})
$$

Over $\mathrm{D}^{*}:=\mathrm{D} \ominus \tau_{0}$ we have a family $\mathcal{M}_{\mathrm{D}^{*}} \rightarrow \mathrm{D}^{*}$ of $\mathrm{CY}$ manifolds. We will assume that $\mathrm{D}$ is the unit disk and $\tau_{0}$ is the origin of the disk. We know from the theory of Hodge structures that if $\left\{\gamma_{1}, \ldots, \gamma_{b_{n}}\right\}$ is a basis of $H^{n}(\mathrm{M}, \mathbb{Z}) /$ Tor, then the functions:

$$
\left(\ldots, \int_{\gamma_{i}} \omega_{\tau}, \ldots\right)
$$

for $0<|\tau|<1$ and $0<\arg (\tau)<2 \pi$ are solutions of a differential equation with regular singularities. From the fact that the solutions of any differential equation with regular singularities has a logarithmic growth and

$$
h\left(\omega_{\tau}, \omega_{\tau}\right)=\left(\ldots, \int_{\gamma_{i}} \omega_{\tau}, \ldots\right)\left(<\gamma_{i}, \gamma_{j}>\right)\left(\ldots, \int_{\gamma_{i}} \overline{\omega_{\tau}}, \ldots\right)^{t}
$$

we deduce that

$$
h\left(\omega_{\tau}, \omega_{\tau}\right) \leq C\left(\sum_{i=1}^{k} \log \left|\tau_{i}\right|\right)^{2 m} .
$$

On the other hand it is not difficult to see that we have the following expression for

$$
\left(\ldots, \int_{\gamma_{i}} \omega_{\tau}, \ldots\right)=\left(\ldots, \sum_{k=0, \alpha_{k}>0}^{n} a_{k, \alpha_{k}} z^{\alpha_{k}}\left(\log \frac{1}{z}\right)^{k}, \ldots\right)
$$

From here we conclude that the form $\partial(\log (h))$ also has a logarithmic growth. Our theorem is proved. 


\subsection{Construction of the Analogue of the Dedekind $\eta$ Function for Odd Dimensional CY manifolds}

In this paragraph we will construct a holomorphic section $\eta^{N}$ of some power of the dual of the determinant line bundle for any odd dimensional CY manifold. I do not know if the Quillen norm $\left\|\eta^{N}\right\|_{Q}^{2}$ will be $\operatorname{det}\left(\Delta_{0}\right)^{2 N}$ in case the dimension of $\mathrm{M}$ is greater than 3 .

Kazhdan proved that for any arithmetic groups $G$ of rank $\geq 2$ the abelian group $G /[G, G]$ is finite. See [9]. According to Sullivan, the subgroup $\Gamma$ of the mapping class group $\Gamma(\mathrm{M})$ constructed in Section 3.2 is an arithmetic group of rank $\geq 2$

Theorem 57 Let $M$ be an odd dimensional $C Y$ manifold. Let $N=\# \Gamma /[\Gamma, \Gamma]$. Then $\mathcal{L}^{\otimes N}$ is a trivial complex analytic line bundle over $\mathcal{M}(M)$.

Proof: According to Theorem 42

$$
\mathcal{L} \approx R^{0} \pi_{*}\left(\Omega_{\mathcal{X} / \mathcal{M}(\mathrm{M})}^{2 n+1,0}\right),
$$

where $\operatorname{dim}_{\mathbb{C}} M=2 n+1$. Therefore, $\mathcal{L}$ is a subbundle of the flat vector bundle $R^{2 n+1} \pi_{*} \mathbb{C}$, where $\mathbb{C}$ is the locally constant sheaf on $\mathcal{X}$, and

$$
\pi: \mathcal{X} \rightarrow \mathcal{M}(\mathrm{M})
$$

is the versal family of CY manifolds over $\mathcal{M}(\mathrm{M})$. We know from Theorem 31 that

$$
\mathcal{M}(\mathrm{M})=\mathcal{T}(\mathrm{M}) / \Gamma
$$

where $\mathcal{T}(\mathrm{M})$ is the Teichmüller space and $\Gamma$ is the subgroup of of the mapping class group $\Gamma(\mathrm{M})$ constructed in Section 3.2. According to [39], $\Gamma$ is an arithmetic group.

If we lift the flat bundle $R^{n} \pi_{*} \mathbb{C}$ on $\mathcal{T}(\mathrm{M})$, then $R^{2 n+1} \pi_{*} \mathbb{C}$ will be the trivial bundle, i.e.

$$
R^{2 n+1} \pi_{*} \mathbb{C} \approx \mathcal{T}(\mathrm{M}) \times H^{2 n+1}\left(\mathrm{M}_{0}, \mathbb{C}\right)
$$

Let us denote by

$$
\sigma: \mathcal{T}(\mathrm{M}) \rightarrow \mathcal{M}(\mathrm{M})=\mathcal{T}(\mathrm{M}) / \Gamma
$$

the natural projection map. Clearly $\sigma^{*}(\mathcal{L})$ will be a flat complex analytic subbundle of the trivial bundle $\mathcal{T}(\mathrm{M}) \times H^{2 n+1}\left(\mathrm{M}_{0}, \mathbb{C}\right)$.

Proposition 58 Let $N$ be a quasi-projective variety, $\mathcal{E} \approx \mathbb{C}^{n} \times N$ a trivial bundle and $\mathcal{L}$ a flat line bundle over $N$ such that the dual $\mathcal{L}^{*}$ of $\mathcal{L}$ satisfies

$$
\mathcal{L}^{*} \subset \mathcal{E}
$$

then $\mathcal{L}$ is also trivial.

Proof: The proof of Proposition 58 is obvious. 
Proposition 58 implies we that $\sigma^{*}(\mathcal{L})$ will be a trivial line bundle. So we get that

$$
\mathcal{L} \approx \mathbb{C} \times \mathcal{T}(\mathrm{M}) / \Gamma
$$

where $\Gamma$ acts in a natural way on the Teichmüller space and it acts by a character

$$
\chi \in \operatorname{Hom}\left(\Gamma, \mathbb{C}_{1}^{*}\right) \approx \operatorname{Hom}\left(\Gamma /[\Gamma, \Gamma], \mathbb{C}_{1}^{*}\right)
$$

of the group $\Gamma$ on the fibre $\mathbb{C}$. A Theorem of Kazhdan states that $\Gamma /[\Gamma, \Gamma]$ is a finite group if the rank of $\Gamma$ is bigger or equal to 2 . For $C Y$ manifolds $\Gamma$ is an arithmetic group of rank $\geq 2$ according to [39]. From here we deduce that $\mathcal{L}^{N}$ will be a trivial bundle on $\mathcal{M}(\mathrm{M})$, where

$$
N=\# \Gamma /[\Gamma, \Gamma] \text {. }
$$

Theorem 57 is proved.

Definition 59 We will define $\mathfrak{D}_{\infty}$ as follows: Let

$$
\mathfrak{D}:=\overline{\mathcal{M}(M)} \ominus \mathcal{M}(M)
$$

be the discriminant locus, then a point $\tau_{\infty}$ is in $\mathfrak{D}_{\infty}$ if around $\tau_{\infty}$ we can find a disk $\mathcal{D}$ such that

$$
\tau_{\infty} \in \mathcal{D}, \mathcal{D} \ominus \tau_{\infty} \subset \mathcal{M}(M)
$$

and over $\mathcal{D} \ominus \tau_{\infty}$ the family of polarized $C Y$ manifolds has a monodromy group of infinite order in $H_{n}\left(M_{\tau}, \mathbb{Q}\right)$.

Theorem 60 Let $M$ be an odd dimensional $C Y$ and $\operatorname{dim}_{\mathbb{C}} M \geq 3$. Let $N$ be defined as in (8.1). There exists a holomorphic section $\eta^{N}$ of $\left(\mathcal{L}^{*}\right)^{\otimes N}$ such that it can be prolonged to a holomorphic section $\overline{\eta^{N}}$ of the line bundle $\left(\overline{\mathcal{L}^{*}}\right)^{\otimes N}$ such that for each point $m \in \mathcal{M}(M), \eta^{N}(m) \neq 0$, i.e. the support of the zero set of $\overline{\eta^{N}}$ is contained in the support of the divisor $\mathfrak{D}$ or it is equal to it.

Proof: Let $\mathfrak{D}=\bigcup_{i} D_{i}$ be the decomposition of the divisor $\mathfrak{D}$ on irreducible components. Theorem 57 implies that the line bundle $\left(\mathcal{L}^{*}\right)^{\otimes N}$ is holomorphic trivial bundle on $\mathcal{M}(\mathrm{M}) . N$ is defined as in (8.1). So we can conclude that

$$
\left(\overline{\mathcal{L}^{*}}\right)^{\otimes N} \approx \mathcal{O}_{\overline{\mathcal{M}(\mathrm{M})}}\left(\sum_{j} k_{j} D_{j}\right),
$$

where $D_{j}$ are the components of $\mathfrak{D}$. We will prove that the multiplicities $k_{i}$ are non negative integers. Indeed we know from Theorem 56 that the metric defined by formula (7.1) on the line bundle $\mathcal{L}$ is a good one in the sense of Mumford. 
So the Chern form $c_{1}\left(\mathcal{L}^{*}, \mathrm{~h}\right)$ of the good metric $\mathrm{h}$ defined by $(7.1)$ is a positive current on $\overline{\mathcal{M}(\mathrm{M})}$. The Poincaré dual of the cohomology of the current

$$
\left[c_{1}\left(\mathcal{L}^{*}, \mathrm{~h}\right)\right] \in H^{2}(\overline{\mathcal{M}(\mathrm{M})}, \mathbb{Z})
$$

is

$$
\mathcal{P}\left(\left[c_{1}\left(\mathcal{L}^{*}, \mathrm{~h}\right)\right]\right)=\sum_{j} k_{j}\left[D_{j}\right] \in H_{2 n-2}(\overline{\mathcal{M}(\mathrm{M})}, \mathbb{Z})
$$

where the coefficients $k_{i}$ are defined as in (8.2). The positivity of the current $c_{1}\left(\mathcal{L}^{*}, h\right)$ implies that its Poincaré dual current

$$
\sum_{j} k_{j}\left[D_{j}\right]
$$

is positive. From here we can conclude that the coefficients $k_{i}$ are positive integers. Indeed, let $\left[\omega_{D_{i}}\right] \in H^{2 n-2}(\mathrm{M}, \mathbb{Z})$ be such classes of cohomology that:

$$
\int_{D_{j}}\left[\omega_{D_{i}}\right]=\delta_{i j} .
$$

Since the current $\sum_{j} k_{i}\left[D_{j}\right]$ is positive, (8.4) implies

$$
\left\langle\sum_{j} k_{i}\left[D_{j}\right],\left[\omega_{D_{i}}\right]\right\rangle=k_{i} \geq 0
$$

Theorem 60 follows from (8.5).

Corollary 61 The zero set $\left(\overline{\eta^{N}}\right)_{0}$ of the section $\overline{\eta^{N}}$ of the line bundle $\left(\overline{\mathcal{L}^{*}}\right)^{\otimes N}$ is a nonzero divisor such that

$$
\operatorname{Supp}\left(\mathfrak{D}_{\infty}\right) \subseteq \operatorname{Supp}\left(\left(\overline{\eta^{N}}\right)_{0}\right) \subseteq \operatorname{Supp}(\mathfrak{D}) .
$$

Proof: According to Theorem 56 the $L^{2}$ metric $h$ on the relative dualizing sheaf $R^{0} \pi_{*} \Omega_{\mathcal{X} / \mathcal{M}(\mathrm{M})}^{n, 0}$ had logarithmic singularities. So from here we can conclude that the section $\overline{\eta^{N}}$ constructed in Theorem 60 had a finite $L^{2}$ norm, i.e.

$$
\left\|\overline{\eta^{N}}\right\|^{2} \leq c_{0}
$$

Let $\mathcal{U}_{\tau_{\infty}}$ will be an open set containing the point $\tau_{\infty} \in \mathfrak{D}_{\infty}$. From Definition 59 it follows that the monodromy around the point $\tau_{\infty} \in \mathfrak{D}_{\infty}$ will be of 
infinite order. From here we can conclude that the $L^{2}$ metric on the relative dualizing sheaf has a logarithmic growth and so

$$
\lim _{\tau \rightarrow \tau_{\infty}}\left|\frac{\left\langle\omega_{\tau}, \omega_{\tau}\right\rangle}{k \log \left|\tau-\tau_{\infty}\right|}\right|=c<\infty
$$

for some $k>0$ which depends on the index of nilpotency of the monodromy. From the explicit expression for the $L^{2}$ norm of the section $\overline{\eta^{N}}$ we see that

$$
\left.\left\|\overline{\eta^{N}}\right\|^{2}\right|_{\mathcal{U}_{\tau}}=<\omega_{\tau}, \omega_{\tau}>|f(\tau)|^{2} \leq c_{0}
$$

So from (8.8) and (8.9) we can conclude that

$$
\lim _{\tau \rightarrow \tau_{\infty}} f(\tau)=0
$$

From (59) we deduce (8.6) . Corollary 61 is proved.

Remark 62 It is not difficult to prove that in the case of odd dimensional $C Y$ manifolds if $\tau$ is a point in $\overline{\mathcal{M}(M)}$ around which we can find one parameter family of polarized $C Y$ manifolds whose monodromy operator acting on the middle homology is of finite order then

$$
\eta^{N}(\tau) \neq 0
$$

\section{CY Threefolds}

Theorem 63 Let $M$ be a three dimensional $C Y$ manifold and let $N=$ $\# \Gamma /[\Gamma, \Gamma]$. Then there exists a section $\eta^{N}$ of the line bundle $\overline{\left(\mathcal{L}^{*}\right)^{\otimes N}}$ such that the norm of $\eta^{N}$ with respect to the $N$ tensor power of the $L^{2}$ metric on $\mathcal{L}^{*}$ is given by:

$$
\left\|\eta^{N}\right\|_{L^{2}}^{2}=\exp (-N I(M))=\left(\operatorname{det}\left(\Delta_{0}\right)\right)^{N} .
$$

The zero set of $\eta^{N}$ is a nonzero effective divisor whose support contains or is equal to the support of $\mathfrak{D}_{\infty}$, where $\mathfrak{D}_{\infty}$ is defined in Definition 59.

Proof: We know from Theorem 46 that the Quillen norm of the section $\operatorname{det}(\bar{\partial})$ of the determinant line bundle $\mathcal{L}$ on $\mathcal{M}(\mathrm{M})$ is equal to the exponential of the holomorphic Ray-Singer analytic torsion I(M). So

$$
\left\|\operatorname{det}(\bar{\partial})^{*}\right\|_{Q}^{2}=\exp (-\mathrm{I}(\mathrm{M})) \text {. }
$$

On the other hand, we proved in [47] that

$$
\exp (-\mathrm{I}(\mathrm{M}))=\left(\operatorname{det}\left(\Delta_{0}\right)\right) \leq c_{0}
$$


This fact, combined with Theorems 48 and 47, imply that locally on $\mathcal{M}(\mathrm{M})$ the following formula is true:

$$
d d^{c}\left(\log \left(\frac{\left\|\operatorname{det}(\bar{\partial})^{*}\right\|_{Q}^{2}}{\left\langle\omega_{\tau}, \omega_{\tau}\right\rangle}\right)\right)=d d^{c}\left(\log \left(\frac{\operatorname{det}\left(\triangle_{0}\right)}{\left\langle\omega_{\tau}, \omega_{\tau}\right\rangle}\right)\right)=0 .
$$

From here we deduce in [47] that for each point $\tau \in \mathcal{M}(\mathrm{M})$ there exists an open set $\mathcal{U}_{\tau}$ such that

$$
\tau \in \mathcal{U}_{\tau}
$$

and we have

$$
\left\|\operatorname{det}(\bar{\partial})^{*}\left|\mathcal{U}_{\tau} \|_{Q}^{2}=\exp (-\mathrm{I}(\mathrm{M}))\right|_{\mathcal{U}}=\left\langle\omega_{\tau}, \omega_{\tau}\right\rangle\left|f_{\mathcal{U}_{\tau}}(\tau)\right|^{2},\right.
$$

where $f_{\mathcal{U}_{\tau}}(\tau)$ is a holomorphic function in $\mathcal{U}_{\tau}$.

Proposition 64 Let us choose $\mathcal{U}$ such that $\mathcal{U} \cap \mathfrak{D}_{\infty} \neq \emptyset$. Let

$$
\tau_{\infty} \in \mathfrak{D}_{\infty} \cap \mathcal{U}
$$

Let $f_{\mathcal{U}}(\tau)$ be the holomorphic function defined by (9.1) in $\mathcal{U} \ominus \mathcal{U} \cap \mathfrak{D}_{\infty}$. Then $f_{\mathcal{U}}$ is well defined at the point

$$
\tau_{\infty} \in \mathfrak{D}_{\infty}=\overline{\mathcal{M}(M)} \ominus \mathcal{M}(M)
$$

and $f_{\mathcal{U}}\left(\tau_{\infty}\right)=0$.

Proof: Indeed, we proved in Theorem 56 that $\left\langle\omega_{\tau}, \omega_{\tau}\right\rangle$ have a logarithmic growth around $\tau_{\infty} \in \mathfrak{D}_{\infty}$. This follows from the definition of $\mathfrak{D}_{\infty}$. Theorem 49 implies that

$$
\left\|\operatorname{det}(\bar{\partial})^{*}\right\|_{Q}^{2}=\exp (-\mathrm{I}(\mathrm{M}))=\left(\operatorname{det}\left(\triangle_{0}\right)\right) \leq c_{0}
$$

if the complex dimension of $\mathrm{M}$ is three. We know from (9.1) that

$$
\left.\exp (-\mathrm{I}(\mathrm{M}))\right|_{\mathcal{U}}=<\omega_{\tau}, \omega_{\tau}>\left|f_{\mathcal{U}}(\tau)\right|^{2} \leq c_{0}
$$

So by using the same arguments as in the proof of Corollary 61, we can conclude that

$$
\lim _{\tau \rightarrow \tau_{0}} f_{\mathcal{U}}\left(\tau_{\infty}\right)=0
$$

From (9.3) we deduce that $f_{\mathcal{U}}$ can be continued analytically around any point

$$
\tau_{\infty} \in \mathfrak{D}=\overline{\mathcal{M}(\mathrm{M})} \ominus \mathcal{M}(\mathrm{M}) .
$$

Proposition 64 is proved. 
From (9.2) we can conclude:

Corollary 65 The zero set $\mathfrak{D}_{I(M)}$ of the function $\exp (-I(M))$ is a nonzero effective divisor such that

$$
\text { Supp } \mathfrak{D}_{\infty} \subseteq \operatorname{Supp} \mathfrak{D}_{I(M)} \subseteq \operatorname{Supp} \mathfrak{D}_{I(M)}
$$

Proposition 64 and Corollary 65 imply Theorem 63. Let the complex analytic functions $f_{\mathcal{U}}$ be defined by (9.1). According to Proposition 64 the functions $f_{\mathcal{U}}$ vanish exactly where $\exp (-\mathrm{I}(\mathrm{M}))$ is zero. So we can conclude that the zero set of $\exp (-\mathrm{I}(\mathrm{M}))$ is an effective divisor $\mathfrak{D}_{\mathrm{I}(\mathrm{M})}$ such that

$$
\text { Supp } \mathfrak{D}_{\infty} \subseteq \operatorname{Supp} \mathfrak{D}_{\mathrm{I}(\mathrm{M})} \subseteq \operatorname{Supp} \mathfrak{D}
$$

where

$$
\mathfrak{D}=\overline{\mathcal{M}(\mathrm{M})} \ominus \mathcal{M}(M) .
$$

So the zero set of the complex analytic functions $f_{\mathcal{U}}$ is exactly the divisor $\mathfrak{D}_{\mathrm{I}(\mathrm{M})} \cap \mathcal{U}$. From 9.1 we derive that on open sets $\mathcal{U}$ and $\mathcal{V}$ such that

$$
\mathcal{U} \cap \mathfrak{D}_{\infty} \neq \emptyset \quad \text { and } \quad \mathcal{V} \cap \mathfrak{D}_{\infty} \neq \emptyset
$$

we have on $\mathcal{U} \cap \mathcal{V}$

$$
\left|f_{\mathcal{U}}\right|^{2}=\left|\sigma_{\mathcal{U V}}\right|^{2}\left|f_{\mathcal{V}}\right|^{2}
$$

where $\sigma_{\mathcal{U V}}$ are the transition functions that define the line bundle $\overline{\mathcal{L}^{*}}$.

From Theorem 57 we know that the line bundle $\left(\mathcal{L}^{*}\right)^{\otimes N}$ is a trivial holomorphic one on $\mathcal{M}(\mathrm{M})$ where

$$
N=\#(\Gamma /[\Gamma, \Gamma]) .
$$

From this fact and (9.5) we deduce that if you fix a covering $\left\{\mathcal{U}_{i}\right\}$ of $\overline{\mathcal{M}(\mathrm{M})}$ then the functions $\left\{\left(f_{\mathcal{U}_{i}}\right)^{N}\right\}$ define a global section $\overline{\eta^{N}}$ of $\overline{\left(\mathcal{L}^{*}\right)}{ }^{\otimes N}$.

The equation (9.1) implies that the support of the zero set $\left(\overline{\eta^{N}}\right)_{0}$ of the section $\overline{\eta^{N}}$ is the same as of the support of the zero set $\mathfrak{D}_{\mathrm{I}(\mathrm{M})}$ of $\exp \left(-\mathrm{I}\left(\mathrm{M}_{\tau}\right)\right)$, i.e.

$$
\operatorname{Supp}\left(\overline{\eta^{N}}\right)_{0} \equiv \operatorname{Supp} \mathfrak{D}_{\mathrm{I}(\mathrm{M})}
$$

Combining (9.4) and (9.6), we get that

$$
\operatorname{Supp}\left(\mathfrak{D}_{\infty}\right) \subseteq \operatorname{Supp}\left(\overline{\eta^{N}}\right)_{0} \subseteq \operatorname{Supp}(\mathfrak{D}) .
$$

If we prove that the effective divisor $\left(\overline{\eta^{N}}\right)_{0}$ is nonzero then Theorem 63 will be proved. 
Proposition $66\left(\overline{\eta^{N}}\right)_{0}$ is a nonzero divisor.

Proof: Since the $L^{2}$ metric on the line bundle $\mathcal{L}^{*}$ defines a Chern form which is exactly the imaginary part of the Weil-Petersson metric and by (9.6), we can conclude that the support $\operatorname{Supp}\left(\overline{\operatorname{det}(\bar{\partial})^{*}}\right)$ of the section $\overline{\operatorname{det}(\bar{\partial})^{*}}$ will be a nonzero divisor. This is so since by combining (9.6), (9.2) and (9.5) we can conclude that the line bundle $\left(\overline{\mathcal{L}^{*}}\right)^{\otimes N}$ has a non holomorphic section $\eta^{N}$ whose zero set $\left(\overline{\eta^{N}}\right)_{0}$ is a nonzero effective divisor.

Theorem 63 is proved.

Corollary 67 There exists a multi-valued holomorphic section $\eta$ of the dual of the determinant line bundle $\mathcal{L}$ over $\mathcal{M}(M)$ such that the norm of $\eta$ with respect to the metric defined in Theorem 56 is equal to $\exp (-I(M))$.

\section{Some Problems}

Problem 68 Can one find a product formula for $\eta$ around points of maximal degenerations, which would mean that around such points the monodromy operator has an index of unipotency $n+1$ ?

For a more precise discussion of Problem 68, see [48]. Problem 68 is closely related to paper [4] and more precisely to the counting problem of elliptic curves on the CY threefold.

Problem 69 Is is true that any CY manifold can be deformed to an algebraic manifold with one conic singularity?

Problem 69 is related to the following problem: Let $M$ be an algebraic variety embedded in $\mathbb{P}^{N}$. Let $\mathcal{H}_{\mathrm{M} / \mathbb{P}^{N}}$ be the component of the Hilbert scheme that contains the point that corresponds to $\mathrm{M}$. Let $\mathfrak{D}_{\mathrm{M}}$ be the set of the points in $\mathcal{H}_{\mathrm{M} / \mathbb{P}^{N}}$ that correspond to singular varieties. It is not difficult to prove that $\mathfrak{D}_{\mathrm{M}}$ is a closed subvariety in $\mathcal{H}_{\mathrm{M} / \mathbb{P}^{N}}$.

Problem 70 Suppose that $\mathfrak{D}_{M}$ is a divisor in $\mathcal{H}_{M / \mathbb{P}^{N}}$. Is it true that the generic point of $\mathfrak{D}_{M}$ corresponds to a projective manifold with only one conic singularity?

The results of this paper suggest that one can expect $\mathfrak{D}_{\mathrm{M}}$ to be a divisor in $\mathcal{H}_{\mathrm{M} / \mathbb{P}^{N}}$ when $\mathrm{M}$ is an odd dimensional $\mathrm{CY}$ manifold $\mathrm{M}$. Problem 70 is closely related to Miles Ried's conjecture that the moduli spaces of all CY threefolds are connected.

Problem 71 In Theorem 60 we constructed a holomorphic section $\bar{\eta}^{N}$ of the prolonged line bundle $\left(\overline{\mathcal{L}^{*}}\right)^{\otimes N}$ for any odd dimension. It is natural to expect that the $L^{2}$ norm of $\bar{\eta}^{N}$ to be $\operatorname{det}\left(\Delta_{0}\right)^{2 N}$. 


\section{References}

[1] Abramovich, D., J.-F. Burnol, J. Kramer and C. Soulé: Lectures on Arakelov Geometry. Cambridge Studies In Advanced Mathematics 33, Cambridge University Press, Cambridge, 1992.

[2] Aspinwall, P. And D. Morrison: Chiral Rings do not Suffice: $N=(2,2)$ theories with non zero fundamental group, Phys. Lett. B 334 (1994), 79-86.

[3] Berline, N., E. Getzler and M. Vergne: Heat Kernels and Dirac Operators, Springer Verlag, 1991.

[4] Bershadsky, M., S. Cecotti, H. Ooguri and C. Vafa: KodairaSpencer Theory of Gravity and Exact Results for Quantum String Amplitude, Comm. Math. Phys. 165 (1994), 311-428.

[5] Bismut, J.-M. And D. Freed: The Analysis of Elliptic Families I. Metrics and Connections on Determinant Line Bundles, Comm. Math. Phys. 106 (1986), 159-176.

[6] Bismut, J.-M., H. Gillet And C. Soulé: Analytic Torsion and Holomorphic Determinant Bundles I. Bott-Chern Forms and Analytic Torsion, Comm. Math. Phys. 115 (1998), 49-78.

[7] Bismut, J.-M., H. Gillet and C. Soulé: Analytic Torsion and Holomorphic Determinant Bundles II. Direct Images and Bott-Chern Forms, Comm. Math. Phys. 115 (1988), 79-126.

[8] Bryant, R. and P. Griffiths: Some Observations on the Infinitesimal Period Relations for Regular Threefolds with Trivial Canonical Bundle. In Arithmetic and geometry, Vol. II, 77-102. Progr. Math. 36, Birkhäuser Boston, Boston, MA, 1983.

[9] Delaroche, Cl. and A. A. Kirillov: Sur les Relations entre l'espace dual d'un groupe et la structure de ses sous-groupes fermés" (d'après D. A. Kazhdan). Séminaire Bourbaki 10, Exp. No. 343, 507-528. Soc. Math. France, Paris, 1995.

[10] Demailly, J.-P.: $L^{2}$ vanishing theorems for positive line bundles and adjunction theory. In Transcendental methods in algebraic geometry (Cetraro, 1994), 1-97. Lecture Notes in Math. 1646, Springer, Berlin, 1996.

[11] Grothendieck, A.: Éléments de Géométrie Algébrique. Inst. Hautes Études Sci. Publ. Math. 8, 1961.

[12] Grothendieck, A.: Séminaire de Géométrie Algébrique. Inst. Hautes Études Sci., Paris, 1960-1961.

[13] Griffiths, Ph.: Periods of Integrals on Algebraic Manifolds I and II. Amer. J. Math. 90 (1968), 568-626 and 805-865.

[14] Griffiths, Ph.: Periods of Integrals on Algebraic Manifolds III. Inst. Hautes Études Sci. Publ. Math. 38 (1970), 125-180. 
[15] Griffiths, Ph. And J. Harris: Principles of Algebraic Geometry. John Wiley \& Sons, New York, 1978.

[16] Gelfand, I. M., M. Kapranov and A. Zelevinsky: Discriminants, Resultants and Multidimensional Deeterminants. Birkhäuser, 1994.

[17] Jorgenson, J. And A. Todorov: Analytic discriminants for manifolds with canonical class zero. In Manifolds and geometry (Pisa, 1993) , 223-260, Sympos. Math. 36, Cambridge Univ. Press, Cambridge, 1996.

[18] Jorgenson, J. And A. Todorov: An analytic discriminant for polarized algebraic K3 surfaces. In Mirror symmetry III (Montreal, PQ, 1995), 211-261. AMS/IP Stud. Adv. Math. 10, Amer. Math. Soc., Providence, RI, 1999.

[19] Helgason, S.: Differential Geometry, Lie Groups, and Symmetric Spaces. Pure and Applied Mathematics 80. Academic Press Inc., New YorkLondon, 1978.

[20] Hörmänder, L.: Introduction to Complex Analysis in Several Variables. D. Van Nostrand Company, Inc., Princeton, Toronto, London, 1966.

[21] Kodaira, K. And J. Morrow: Complex Manifolds. Holt, Rinehart and Winston, Inc., New York-Montreal-London, 1971.

[22] Kulikov, V.: Degenerations of K3 surfaces and Enriques Surfaces. Izv. Akad. Nauk USSR Ser. Mat. 41 (1977), no. 5, 1008-1042.

[23] LAng, S.: Differential and Riemannian Manifolds. Third edition. Graduate Texts in Mathematics 160. Springer-Verlag, New York, 1995.

[24] Liu, K., A. Todorov, S.-T. Yau And K. Zuo: Shafarevich's Conjecture for CY Manifolds I, preprint 2002.

[25] Liu, K., A. Todorov, S.-T. YAu And K. Zuo: Shafarevich's Conjecture for CY Manifolds II, preprint 2002.

[26] Lu, Z.: On the Hodge Metric of the Universal Deformation Space of CY Threefolds. J. Geom. Anal. 11 (2001), no. 1, 103-118.

[27] Lu, Z.: On the Curvature Tensor of the Hodge Metric of the Moduli Space of CY Threefolds. J. Geom. Anal. 11 (2001), no. 4, 635-647.

[28] Lu, Z. And X. Sun: Weil-Petersson Geometry on Moduli Space if Polarized of CY Manifolds, preprint.

[29] Matsusaka, T., D. Mumford: Two Fundamental Theorems on Deformations of Polarized Varieties. Amer. J. Math. 86 (1964), 668-684.

[30] Mumford, D.: Hirzebruch's Proportionality Principle in the NonCompact Case. Inv. Math. 42 (1977), 239-272.

[31] Newlander, A. And L. Nirenberg: Complex Analytic Coordinates in Almost Complex Manifolds. Ann. of Math. 65 (1957), 391-404. 
[32] Palais, R.: On the Existence of Slices for Actions of Non-Compact Lie Groups. Ann. of Math. (2) 73 (1961), 295-323.

[33] Roe, J.: Elliptic Operators, Topology and Asymptotic Methods. Pitman Research Notes in Mathematics Series 179, Longman Scientific \& Technical, 1988 .

[34] Ray, D. And I. Singer: Analytic Torsion for Complex Manifolds. Ann. of Math. 98 (1973), 154-177.

[35] Raghunathan, M. S.: Discrete Subgroups of Lie Groups. Ergebnisse Der Mathematik und Ihrer Grenzgebiete, Band 68, Springer-Verlag, 1972.

[36] Shafarevich, I. R.: Collected mathematical papers. Springer-Verlag, Berlin, 1989.

[37] Siu, Y. T.: Effective Very Ampleness. Inv. Math. 73 (1995), 251-265.

[38] Siu, Y. T. AND S.-T. Yau: Compactification of Negatively Curved Complete Kähler Manifolds of Finite Volume. In Seminar on Differential Geometry, 363-380. Ann. of Math. Stud. 102, Princeton Univ. Press, Princeton, N.J., 1982.

[39] Sullivan, D.: Infinitesimal computations in topology. Inst. Hautes Études Sci. Publ. Math. 47 (1977), 269-331.

[40] Szendroi, B.: On an Example of Aspinwall and Morrison, math. AG/9911064.

[41] Szendroi, B.: Calabi-Yau Threefolds with a Curve of Singularities and Counterexamples to Torelli Problem, math. AG/9901078.

[42] Tian, G.: Smoothness of the Universal Deformation Space of Calabi-Yau Manifolds and its Petersson-Weil Metric. In Mathematical aspects of string theory (San Diego, Calif., 1986), 629-646. Adv. Ser. Math. Phys. 1, World Sci. Publishing, Singapore, 1987.

[43] Tian, G.: Smoothing 3-folds with Trivial Canonical Bundle and Ordinary Double Points. In Essays on Mirror Manifolds (edited by Shing-Tung Yau), 459-479. International Press, 1992.

[44] Todorov, A.: Applications of Kähler-Einstein-Calabi-Yau Metric to Moduli of K3 Surfaces. Inv. Math. 61 (1980), 251-265.

[45] Todorov, A.: The Weil-Petersson Geometry of Moduli Spaces of $\mathrm{SU}(n \geq 3)$ (Calabi-Yau Manifolds) I. Comm. Math. Phys. 126 (1989), $325-346$.

[46] Todorov, A.: Analytic Torsion and the Moduli of Odd Dimensional CY Manifolds I, preprint, 1998.

[47] Todorov, A.: Analytic Torsion and the Moduli of Odd Dimensional CY Manifolds II, preprint. 
[48] Todorov, A.: Analytic Torsion and the Moduli of Odd Dimensional CY Manifolds III, preprint, 1998.

[49] Todorov, A.: The Weil-Petersson Geometry of Moduli of CY Manifolds II, preprint.

[50] Viehweg, E.: Quasi-Projective Moduli for Polarized Manifolds. Ergebnisse der Mathematik und iher Grenzgebiete (3) 30, Springer-Verlag, 1995.

[51] Wang, Chin-Lung: On the Incompleteness of the Weil-Petersson Metric along Degenerations of Calabi-Yau Manifolds. Math. Res. Lett. 4 (1997), no. $1,157-171$.

Recibido: 15 de marzo de 2002

Andrey Todorov

Department of Mathematics

University of California

Santa Cruz, CA 94109, USA

Bulgarian Academy of Sciences

Institute of Mathematics

Sofia, Bulgaria

andreytodorov@msn.com

This paper is dedicated to my good friend José Luis Vicente. 